\title{
Spatial Risk Analysis of Power Systems Resilience During Extreme Events
}

a)

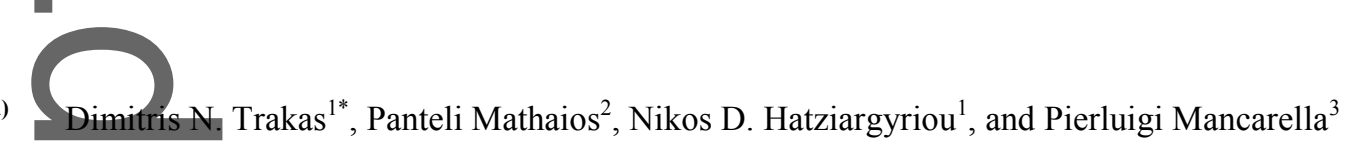

${ }^{1}$ National Technical University of Athens, Greece.

${ }^{2}$ University of Manchester, UK.

${ }^{3}$ University of Manchester, UK, and University of Melbourne, Australia.

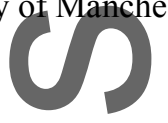

*Address correspendence to Dimitris Trakas, School of Electrical and Computer Engineering, Electric Energy Systems LaboratoryNTUA, Athens, Greece; tel+30 210772 3699; dtrakas@power.ece.ntua.gr

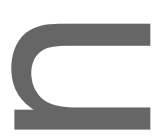

\section{ABSTRACT:}

The increased frequency of extreme events in recent years highlights the emerging need for the development of methods that could contribute to the mitigation of the impact of such events on critical infrastructures, as well as boost their-resilience against them. This paper proposes an online spatial risk analysis capable of providing an indication of the evolving risk of power systems regions subject to extreme events. A Severity Risk Index (SRI) with the support of real-time monitoring assesses the impact of the extreme events on the power system resilience, with application on the effect of windstorms on transmission networks. The index considers the spatial and temporal evolution of the extreme event, system operating conditions and the degraded system performance during the event. SRI is based on probabilistic risk by condensing the probability and impact of possible failure scenarios while the event is spatially moving across a power system. Due to the large number of possible failures during an extreme event, a scenario generation and reduction algorithm is applied in order to reduce the computation time. SRI provides the operator with a probabilistic assessment that could lead to effective resilienee-based decisions for risk mitigation. The IEEE 24-bus reliability test system has been used to demonstrate the effectiveness of the proposed online risk analysis which was embedded in a Sequential Monte

This is the author manuscript accepted for publication and has undergone full peer review but has not been through the copyediting, typesetting, pagination and proofreading process, which may lead to differences between this version and the Version of Record. Please cite this article as doi: 10.1111/risa.13220.

This article is protected by copyright. All rights reserved. 
Carlo simulation for capturing the spatiotemporal effects of extreme events and evaluating the effectiveness of the proposed method.

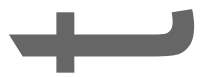

KEY WORDS: Extreme weather event; power system; resilience; resiliency; spatial risk

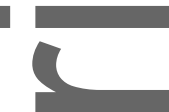

Social Media Abstract

Extreme weather events in recent years highlight the need for the development of methods that could assess the impact of such events on power systems and lead to effective resilience-based decisions for risk mitigation.

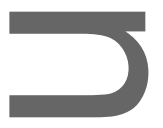

\section{INTRODUCTION}

In the light of climate change and the prevalence of severe weather and natural disasters worldwide, the assessment and enhancement of power systems resilience to such extreme events is becoming of growing concern. In the context of power systems, resilience can be broadly defined as their ability to withstand and minimize the effects of events, recover quickly from these disruptive events and adapt their operation and structure in order to be better prepared for future events (National Infrastructure Advisory Council, 2010; Panteli \& Manearella, 2015c). One of the most distinct characteristics of these events (i.e., extreme weather and natural disasters) is the extent of the impact they might have on a power system. That is, due to the nature of these events, they might spatially spread across a large area, thus affecting large parts of a power system. The development of analysis tools capable of capturing the spatial risk of these large-scale events is therefore critically important. This spatial risk analysis can be performed in two ways, namely online, supporting the short-term decision-making of the system operators for applying the appropriate measures during the event to mitigate its impact, or offline, enabling the long-term strategic planning for boosting power systems resilience to future (similar or unforeseen) events (Ciapessoni et al., 2013).

The impact assessment of extreme weather events on power systems has attracted the interest of many researchers. Many works have focused on the estimation of spatial power outages to distribution system due to extreme weather events. Liu, Davidson, and Apanasovich (2008) developed statistical models for estimating the spatial distribution of power outages due to hurricanes and ice storms. More specifically, a large database of This article is protected by copyright. All rights reserved. 
recent outages experienced by six hurricanes and eight ice storms was used to develop spatial generalized linear mixed models for predicting number of outages in distribution system. Han, Guikema, Quiring, Lee, Davidson, and Rosowsky (2009) proposed a model for forecasting power outages in the Gulf Coast region of US. Data about power outages during nine past hurricanes were used to develop a negative binomial generalized linear model for predicting power outages. Using the same data, Han, Guikema, and Quiring (2009) developed a Poisson generalized additive model that provided better predictions due to its ability to capture the nonlinearity

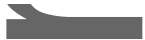
relationship in data. The models proposed by Liu et al. (2008), Han, Guikema, Quiring, Lee, Davidson, and Rosowsky (2009), Han, Guikema, and Quiring (2009) and tree-based data mining techniques were compared to their accuracy of estimating the number of damaged poles due to a hurricane by Guikema, Quiring, and Han (2010). An approach based on classification and regression trees was developed by Quiring, Zhu, and Guikema (2011) to evaluate the impact of soil and elevation data on power outages on the predictive accuracy of power outage models. Similar data to Han, Guikema, Quiring, Lee, Davidson, and Rosowsky (2009) and Han, Guikema, and Quiring (2009) were used by Quiring et al. (2011). Nateghi, Guikema, and Quiring (2014) presented a model for estimating power outages due to hurricanes using less input variables comparing to the aforementioned statistical approaches. Given the difficulty of gathering data of previously exprerienced hurricanes, an important feature of the developed model is the usage of only publicly available data. Guikema et al. (2014) developed a model for predicting hurricane power outages, using also only publicy available data. This model is able to provide accurate estimates and can be used over a wide area. The accuracy of different types of models for prediciting power outages in Connecticut using data from eighty-nine storms of different types and from different seasons to calibrate their models. were examined by Wanik, Anagnostou, Hartman, Frediani, and Astitha (2015) and He et al. (2016) In these works, that predict the power outages before the landfall of an extreme weather, historical data about power outages of previous extreme weather events are required in order to train their models while their effectiveness could be limited due to data non-availability.

Furthermore, various works have developed models that simulate the spatial impact of extreme weather on power systems in order to assess the damage and the risk of power systems. Monte Carlo simulation was used in many works to capture the uncertainty of hurricane characteristics and its impact on power system components. A probabilistic hurricane model for supporting the estimation of hurricane induced damages in Florida was introduced by $\mathrm{Xu}$, and Brown (2008). Utilizing a Monte Carlo simulation, the distribution of simulated peak gusts throughout Florida based on historical hurricanes were generated. Considering a hurricane probability model, a risk assessment approach for evaluating the reliability of a distribution system in Northeast U.S. 
against to windstorms was developed by Li et al. (2014). A Monte Carlo simulation were applied to generate artificial wind storms using historical data and based on weather-dependent models of failure and repair time of system components, reliability indices of the system were computed. Ouyang, and Dueñas-Osorio (2014) and Panteli, and Mancarella (2015b) presented probabilistic models for quantifying the resilience of power systems against extreme weather using the concept of fragility curves and taking into account the full restoration of the power system. In addition, based on the proposed models, Ouyang, and Dueñas-Osorio (2014) and Panteli, and Mancarella (2015b) examined various measures for power system resilience enhancement. A comprehensive review of related previous works, on how to assess the impact of weather on power system resilience, has been presented by Panteli, and Mancarella (2015a). Song, Wang, Xin, and Gan (2013) proposed a risk-based model for dynamic security assessment of power systems under extreme weather events. An optimization problem is solved to apply the appropriate measures in case the failures threat the dynamic security of the system. A risk index was computed taking into account the weather-dependent failure probabilities of transmission line segment and the cost of generation and load shedding that is carried to lead the system to a stable state. Chen, $\mathrm{Wu}$, and Lou (2011) proposed a risk-based method for static security assessment against weather events. Taking into account the probabilities of the failures and their severity an index was computed to assess the risk of power system static security. The severity of the contingencies reflects the voltage decrease and the branch overloading. Chen et al (2011) used fuzzy techniques to model the relationship between failure rates of transmission lines and weather conditions, such as Liu, and Singh (2011) that proposed a model for assessing the impact of a hurricane on the short-term reliability of the power system using the minimal cut-set method. Bhat, and Meliopoulos (2016) presented a model that compute the stress on distribution poles due to wind and asses the probability of a pole to fail. The model takes into account various parameters that affect pole stress, such as the features of the distribution system and of the weather event.

This paper proposes an online risk analysis capable of providing an indication of the evolving risk of power systems subject to large-scale extreme events that might spatially spread across them. The proposed method is based on real-time monitoring and there is no need of historical data of similar weather events. The required data are the fragility curves of the system components that express the failure probability of a component as a function of the weather parameter (e.g. wind or snow density). In this paper, the branches and the towers of the power system are considered as the vulnerable components to an extreme weather event. A Severity Risk Index (SRI) adaptive to the evolving system and event conditions reflecting the probabilistic risk is proposed. $S R I$ condenses the probability of possible failure scenarios and their impact on power system during 
the event and enables the real-time evaluation of the risk introduced to the system. This index, supported by smart grid technologies for monitoring the prevailing system conditions, could be a useful tool for supporting the pre-storm decision-making actions for mitigating the impact on power system. A scenario generation and reduction algorithm was used to support the real-time application of the proposed method when the number of possible failure scenarios was large and the computation of SRI could not be conducted in a reasonable timeframe. Latin Hypercube Sampling (LHS) was used to generate an infinite number of possible failure scenarios and a Backward Reduction Algorithm was used to reduce the number of these scenarios into a computationally tractable size. The scenarios after the application of the reduction algorithm were used to compute SRI, which takes into account their probability and impact on the resilience of the power system. The impact of each scenario was considered equal to the amount of load shedding that was carried out to lead the system to a stable state. A Sequential Monte Carlo simulation was applied to capture the spatiotemporal impacts of the extreme event and validate the effectiveness of the proposed online risk analysis.

The contribution of the paper focuses on the risk-based spatial and temporal estimation of the impact during an extreme event (reflected here by the load shedding occurring due to the event) in order to allow the system operator to apply actions to mitigate this impact and plan which loads to shed in a controllable way for satisfying the steady state constraints such as load generation balance, thermal limits and voltage limits. In case the balance between generation and demand is not achieved, there is the danger of system instability and eventually collapse with very serious consequences. Furthermore, cascading events could be triggered by a number of initiating events leading to system blackout. The major advantages of the proposed method are that (1) is applied to estimate the impact on power system at regular time steps during an extreme weather, (2) is based on reat-time monitoring and there is no need of historical data about the impact of previous extreme weather events on power system, (3) is adaptive to weather conditions and to the constantly changing topology of the network. Furthermore, by applying the proposed scenario generation and reduction algorithms, the estimation of the impact is carried out in a short time and enable the real-time support of mitigation decisions.

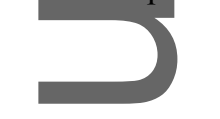

The IEEE 24-Bus Reliability Test System (RTS) (Grigg et al., 1999) has been used to demonstrate the proposed method in case of an extreme windstorm. Different windstorm scenarios with different spatial and intensity (i.e., maximum wind speed) characteristics were used to assess the effectiveness of the designed simulation model. In this case study application, the test network was divided in geographical regions to model 
the spatial impact of windstorms on the transmission system and SRI was computed for each region. Each region was characterized by a specific wind profile and was considered homogenous within the region. The concept of fragility curves was used to obtain the wind-dependent failure probabilities of the power system components. These failure probabilities were used to identify the vulnerable components and draw the possible failure scenarios during a windstorm, which were then fed into the scenario generation and reduction for computing SRI.

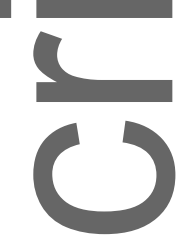

The paper is structured as follows. Section 2 describes the procedure for computing the proposed SRI. In Section 3 the proposed method is illustrated using the IEEE 24-bus Reliability Test System and the results for each region are presented. Section 4 summarizes and concludes the paper.

\section{MODELLING OF SEVERITY RISK INDEX}

SRI reflects the expectation of severity of the extreme event (i.e., severe weather in this case study application) and is defined as the summation of terms, where each term is the product of the probability times the impact for each scenario. The sum goes over the whole set of possible failure scenarios in the next simulation step. In the present work, the duration of the extreme event was divided into equal time intervals and in each interval the $S R I$ was computed for the next one. The computation of the SRI was based on the following steps: (1) the spatial and temporal impact of the weather event on power system components was modelled using fragility curves, (2) the vulnerable components were identified, (3) the possible failure scenarios were constructed considering the vulnerable components, and (4) SRI was computed. In case the computation of SRI in step (4) increased significantly due to the large number of possible failure scenarios, the scenario generation and reduction algorifhm was applied in order to provide in step (4) a manageable size of representative scenarios which were used to compute $S R I$ in a reasonable time. The above procedure was applied in each time interval to compute $S R I$ for the next one. Fig 1. presents the flowchart of the proposed risk analysis, in order to assess the impact of extreme weather event in the next time interval. 


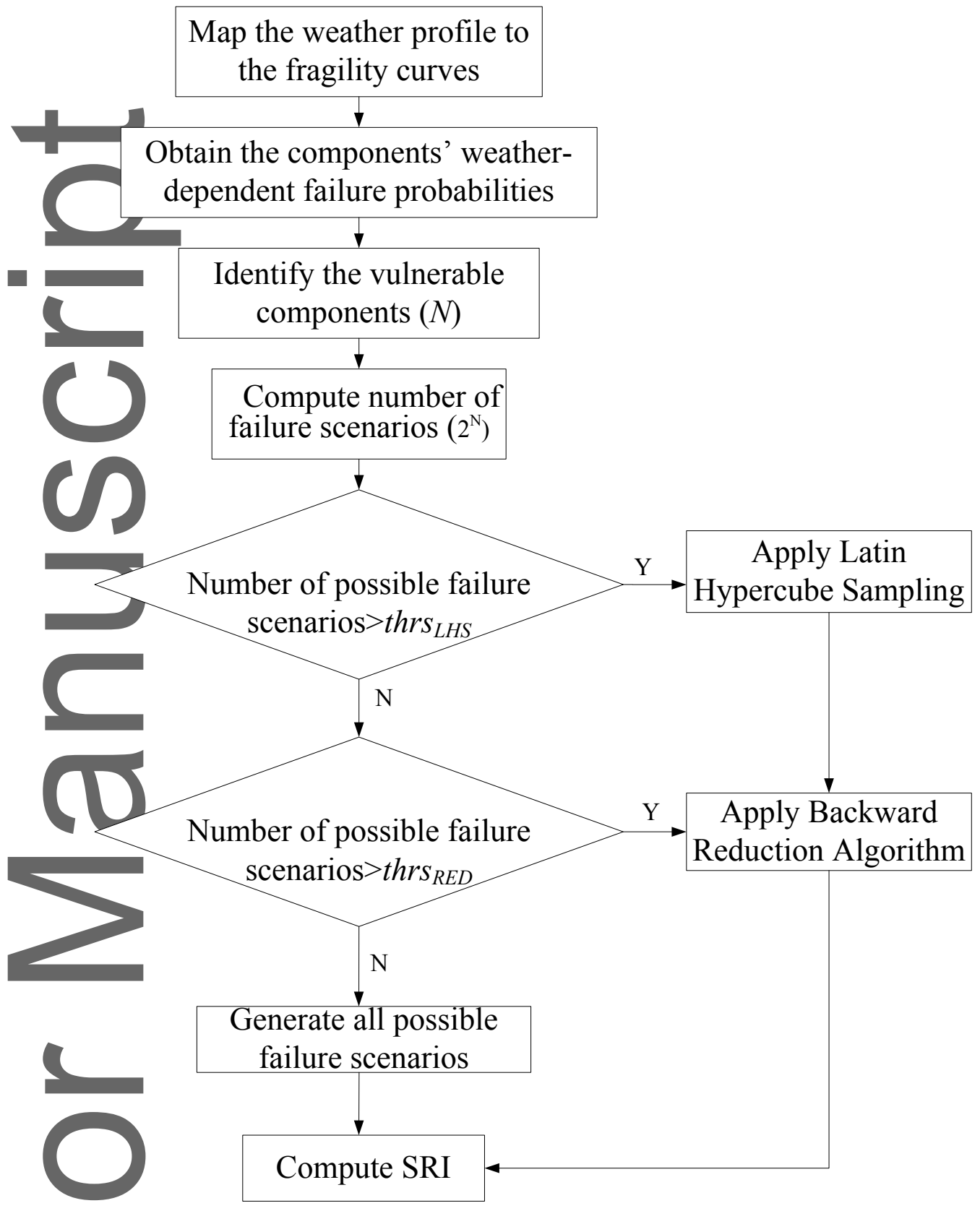

Fig. 1. Generic flowchart of the proposed online risk analysis for computing SRI.

\subsection{Fragility and impact modelling of weather event}

The weather profile of the next simulation step was mapped to the fragility curves for obtaining the weather-dependent failure probabilities of the power system components. Fragility curves are considered an effective tool for relating the failure probabilities of the components to the prevailing weather conditions, with several applications in the research area of the impact modelling of extreme weather and natural disasters. The

This article is protected by copyright. All rights reserved. 
fragility curves that were used in this paper provide the probability of a component to fail as a function of the hourly average wind speed to which the components are exposed. At each hourly simulation step, the probability of a functional component to fail depends only on the hourly average wind speed of that simulation step. These fragility curves were developed in a project funded by Research Council UK (RCUK) based on analytical approaches (Panteli, Pickering, Wilkinson, Dawson, \& Mancarella, 2016). A generic fragility curve is shown in Fig. 2, where two characteristic intensities of the weather parameter can be seen, i.e., $w_{\text {critical }}$ which is the intensity at which the component starts becoming more vulnerable to the weather event (i.e., higher failure probability) and $w_{\text {collapse }}$ the intensity where the failure of the component is certain. The proposed method focuses on the impact of severe windstorms on the branches and towers of the power system.

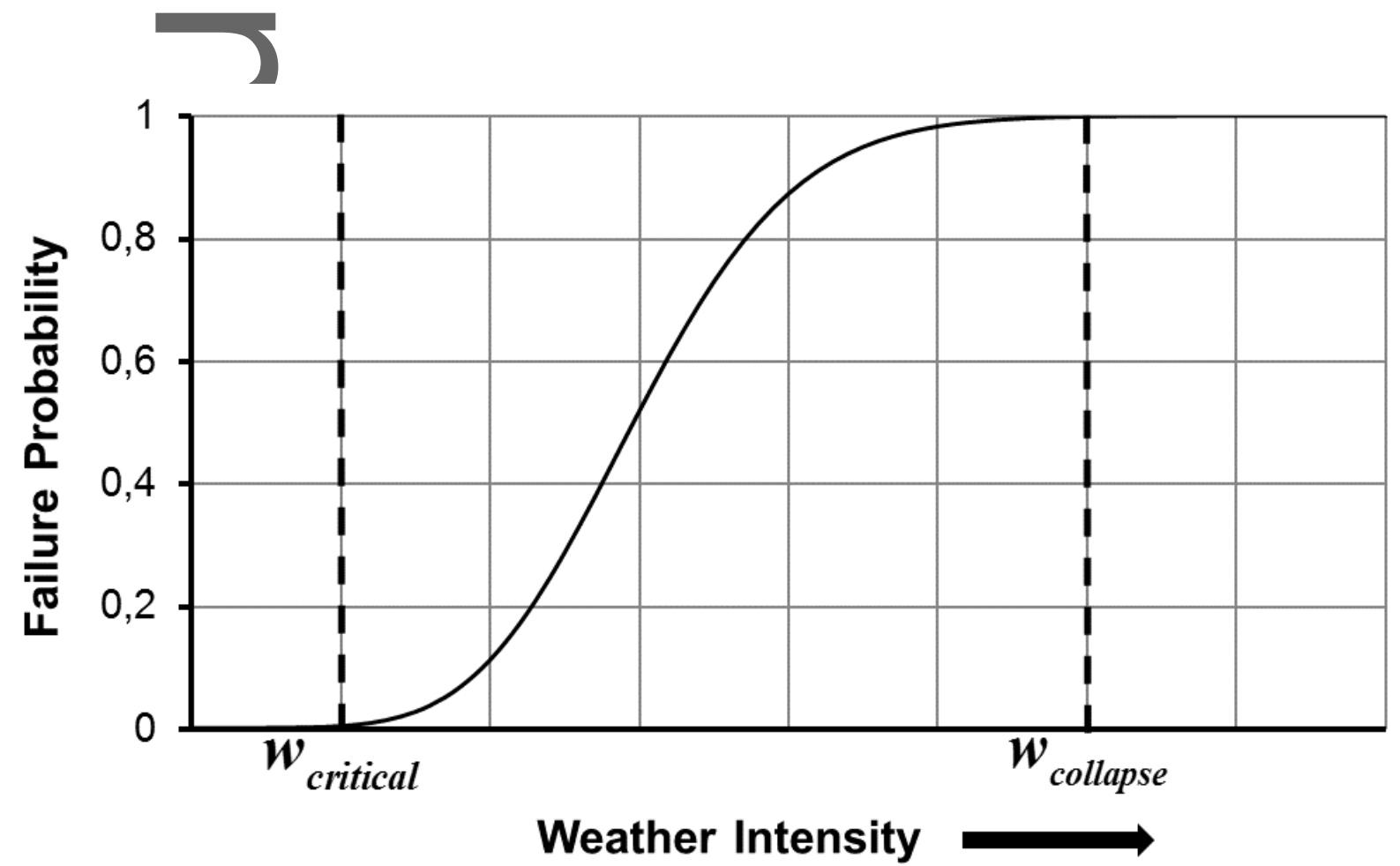

Fig. 2. A generic fragility curve showing the failure probability of a component as a function of the weather intensity.

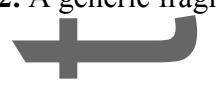

Assuming that the failure of a tower is independent from the failure of the other towers across the same branch, the probability of a branch to fail due to tower trip $P r_{b r, T}$ is defined as:

$$
P r_{b r, T}=1-\left(1-P r_{T}\right)^{N_{T}}
$$

where $\operatorname{Pr}_{T}$ is the individual weather-dependent tower failure probability and is obtained by the tower fragility curve. $N_{T}$ is the number of towers across branch $b r$. The assumption of independent failure probabilities of towers is considered valid. Despite the mechanical forces that are applied to towers in case of an adjacent tower This article is protected by copyright. All rights reserved. 
failure, the collapse of a tower due to these mechanical forces has never been recorded, e.g. in Great Britain (Panteli, Pickering, et al., 2016). Furthermore, according to modelling studies and empirical data from North America, the failure of high voltage transmission tower due to the failure of an adjacent tower is extremely uncommon (Vincent et al., 2004; Eidinger, \& Kempner, 2012).

The overall probability of a branch to trip due to the windstorm is given as follows: (<smiles></smiles>

$$
P r_{b r}=P r_{b r, B}+P r_{b r, T}-P r_{b r, B} P r_{b r, T}
$$

where $P r_{b r, B}$ is the weather-dependent probability of branch $b r$ to trip due to windstorm and is obtained by the branch fragility curve. The overall branch failure probabilities were fed in the next step to identify the vulnerable branches.

It should be noted that in this paper, the impact of high wind speeds on transmission lines and towers was only considered. The impact of other extreme weather disasters, such as floods, was neglected. Synergetic impacts of different weather conditions, such as wind and accumulated rainfall, could be however taken into

$$
10
$$

account using fragility curves that provide the damage probability of a substation vulnerable to floods, as a function of the accumulated rainfall, e.g. Espinoza, Panteli, Mancarella, and Rudnick (2016).

\subsection{Identification of vulnerable components}

The identification of the vulnerable branches was based on the weather-dependent failure probabilities obtained from the fragility curves. The branches with failure probability higher than a pre-specified threshold were labelled as vulnerable. This threshold was selected by the operator and indicates the security criteria to be fulfilled. It is mentioned that by using the fragility curves the probability of a branch to fail depends only on the current wind speed. The stress imposed on branches due to the wind speed of previous hours was not considered for computing the failure probability of the branches.

\subsection{Construction of possible failure scenarios}

Each vutnerable branch could be in functional or damaged state in the next simulation step. The possible failure scenarios were constructed considering all possible combinations of the vulnerable branches. In case of $N$ vulnerable branches, the number of possible failure scenarios is $2^{N}$. The probability $\operatorname{Pr}_{s, k}$ of the $k^{\text {th }}$ possible failure scenario is given as follows: 


$$
P r_{s, k}=\prod_{i=1}^{N_{D}} P r_{b r, i} \prod_{j=1}^{N_{F}}\left(1-P r_{b r, j}\right)
$$

where $P r_{b r, i}$ is the probabilty of the $i^{t h}$ vulnerable branch to be in a damaged state in the next simulation step. $N_{D}$ and $N_{F}$ is the set of damaged and functional branches for the $k^{\text {th }}$ possible failure scenario, resprectively.

\subsection{Computation of $S R I$}

Using the impact and probability of the possible failure scenarios, the $S R I$ of the next simulation step $i$ is defined as:

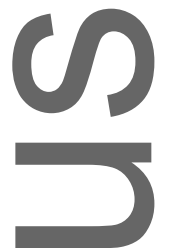

$$
S R I_{i}=\sum_{k=1}^{K} P r_{s, k, i} \cdot I m_{s, k, i}
$$

where $K$ is the set of possible failure scenarios. $P r_{s, k, i}$ and $I m_{s, k, i}$ are the probability and impact of $k^{\text {th }}$ scenario at simulation step $i$, respectively. The impact of each scenario was considered equal to the amount of load shedding (in MWh) that was carried out for leading the system in a stable state. For each scenario, the set of the damaged branches were set out of service and a DC Optimal Power Flow (DC OPF) was executed in order to record the load shedding. The objective function of the DC OPF was to minimize the generation and load shedding cost.

During a severe weather event, the network topology is constantly changing due to branch failures. Realtime monitoring through the use of smart grid technologies, such as Phasor Measurement Units (PMUs), could be used to determine the network topology. In addition, the power system may be divided in islands due to branch failures. Each island was considered as an independent power system. In case of bus isolation, the load shedding that was carried out was equal to the difference between the demand and the bus generation capacity. If the latter was higher than the demand, load shedding was not carried out.

It was assumed that if a branch failed, it was considered as damaged for the rest of the windstorm period (i.e., no restoration takes place during the event) and therefore at each simulation step there might have been already damaged branches due to branch failures in previous simulation steps. As can be seen from the computation steps, SRI takes into consideration the topology of the system, the loading conditions and the weather-dependent failure probabilities of the branches in the next simulation step. Therefore, $S R I$ depends on information that is revealed during the evolution of the weather event and hence is characterized as online. 


\subsection{Scenario generation and reduction algorithm}

During a severe windstorm, a large number of branches may be identified as vulnerable, resulting to a number of possible failure scenarios that may not be computationally tractable for a real-time application. In order to deal with this complexity, a scenario generation and reduction algorithm was embedded in the proposed online risk analysis tool. If the number of possible failure scenarios was higher than a selected threshold $\operatorname{thrs}_{L H S}$, LHS was used to generate a finite large number of scenarios and then the Backward Reduction Algorithm was applied to reduce the number of scenarios in a manageable size thrs $S_{R E D}$. If the number of all possible failure seenarios was lower than $\operatorname{thrS_{LHS}}$ and higher than $\operatorname{thr} S_{R E D}$, only the Backward Reduction Algorithm was applied to reduce the number of scenarios in $t h r s_{R e d}$. In case the number of all possible failure scenarios was lower than $\operatorname{thrs}_{R E D}$, generation and reduction algorithm was not applied and all possible failure scenarios were used in $S R I$ computation

LHS is a stratified-random procedure for generating a finite number of samples for a multidimensional distribution (Mckay, Beckman, \& Conover 2000). The cumulative distribution of each variable (dimension) is divided in $m$ equally probable intervals. A value is randomly picked from each interval and those values are randomly paired in order to produce equally probable scenarios. In this paper, a uniform distribution $U(0,1)$ was considered for each vulnerable branch and was fed in LHS to produce a finite large number of scenarios. Each scenario produced by LHS was a $N$-dimensional vector (for $N$ vulnerable branches) and each element of the vector corresponded to a specific vulnerable branch. The values of the vector were compared with the branch failure probabilities $P r_{b r}$. If a value of the vector was lower than the corresponding branch failure probability, then the branch was considered as damaged; otherwise as a functional one.

A Backward Reduction Algorithm was used to reduce the number of possible failure scenarios to a set of representative scenarios which enable the computation of $S R I$ quickly enough to be considered as a real-time application. In general, the scenario reduction algorithms determine a subset of the initial scenario set and assign new probabilities to the representative scenarios. The problem of optimal reduction of an initial scenario set with finitely many scenarios is defined as finding a reduced scenario subset with fixed cardinality and a probability measure based on this set that has the minimal probability distance from the initial distribution. The probability distance considers the scenario probabilities and the distance of scenario values. The Kantorovich distance $D_{K}$ of probability distributions is used (Heitsch, \& Römisch, 2003; Growe-Kuska, Heitsch, \& Romisch 2003). 
Consider the discrete probability measure $P$ carried by finitely many scenarios $\omega_{i} \in \Omega(i=1, \ldots, S)$ with probabilities $p_{i}, \sum_{i=1}^{S} p_{i}=1 . \omega \in \mathbb{R}^{n}$ is the vector of a scenario belonging to the scenario set $\Omega$. Let $Q$ be another probability measure having scenarios $\omega_{j} \in \Omega^{\prime}$ with probabilities $q_{j}, j=1, \ldots, S^{\prime}$. The Kantorovich distance is given as:

$$
D_{K}(P, Q)=\inf \left\{\begin{array}{c}
\sum_{i=1}^{S} \sum_{j=1}^{S^{\prime}} c\left(\omega_{i}, \omega_{j}\right) \cdot n\left(\omega_{i}, \omega_{j}\right): n\left(\omega_{i}, \omega_{j}\right) \geq 0, \sum_{i=1}^{S} n\left(\omega_{i}, \omega_{j}\right)=q_{j}, \\
\sum_{j=1}^{S^{\prime}} n\left(\omega_{i}, \omega_{j}\right)=p_{i}
\end{array}\right\}
$$

where $n\left(\omega_{i}, \omega_{j}\right)$ retates to the joint probability distributions defined on the domain $\Omega \times \Omega^{\prime} . c\left(\omega_{i}, \omega_{j}\right)$ measures the distance between scenarios which is given by:

$$
c\left(\omega_{i}, \omega_{j}\right):=\left|\omega_{i}-\omega_{j}\right|
$$

where, $|\cdot|$ denotes some norm in $\mathbb{R}^{n}$.

Assume that $S$ is the number of scenarios of the initial entry set in the reduction algorithm and let $J$ denote the set of the deleted scenarios. The optimal choice of the set $J$ with fixed cardinality $\# J$ and $s \in \mathbb{N}, s<S$ is obtained by the optimal reduction problem:

$$
\min \left\{\sum_{u \in J} p_{u} \min _{j \notin J} c\left(\omega_{u}, \omega_{j}\right): J \subset\{1, \ldots, S\}, \# J=S-s\right\}
$$

where $s$ is the number of representative scenarios.

The Backward Reduction algorithm is a heuristic algorithm of the specific case \#J = 1. The algorithm is repeated recursively until the desired number of preserved scenarios has been reached. At each iteration, a scenario $\omega_{l}$ is removed and problem (7) becomes:

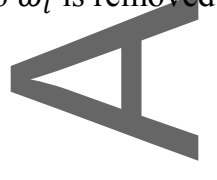

$$
\min _{l \in\{1, \ldots, S\}} p_{l} \min _{j \neq l} c\left(\omega_{l}, \omega_{j}\right)
$$


As can been seen, the deleted scenario is chosen based on its relative closeness to other scenarios and its probability. Therefore, the elimination of a specific scenario means that the probability of this scenario is low and there is a close distance scenario to it. Assume that the minimum is attained for the deleted scenario $\omega_{l_{*}}$ and the scenario $\omega_{j_{*}}$. By deleting the scenario $\omega_{l_{*}}$, the reduced probability measure $Q$ is obtained and $q_{i}=p_{i}$ for all $i \neq l_{*}, j_{*}$ and $q_{j_{*}}=p_{l_{*}}+p_{j_{*}}$.

\section{CASE STUDY APPLICATION}

The IEEE 24-bus Reliability Test System was used as a test network to demonstrate the proposed online risk analysis tool. The method is also applicable to distribution networks provided the relevant data, including the fragility curves of the distribution components, are available. A simulation period of one day (24 hours) and a time resolution of one hour were considered. An adequate number of different time-series wind profiles with varying intensities have been considered as illustrative case scenarios in order to evaluate the effectiveness of the proposed method. It was assumed that the wind speed of the next simulation step (hour) was predicted with a relative error. A real-time hurricane wind forecast model has been proposed by Xie, Bao, Pietrafesa, Foley, and Fuentes (2006). A Sequential Monte Carlo simulation was used in order to evaluate the effectiveness of the proposed method. At every simulation step, the weather-dependent failure probabilities obtained by the fragility curves were compared with a uniformly distributed random number $r \sim U(0,1)$. If the failure probability of the component was larger than $r$, then it was tripped. In this way, the damaged branches by the windstorm at each simulation step were determined. The load shedding due to branches failure was recorded and compared with SRI. Mean Absolute Normalized Error (MANE) was computed to evaluate the performance of the proposed method. Absolute normalized error represents the absolute difference of the recorded load shedding and SRI, expressed as a percentage of the load demand. The SRI and the load shedding provided by Monte Carlo simulation at each hour were recorded. MANE was computed according to:

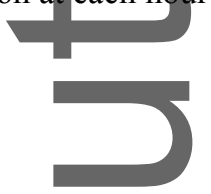

$$
\operatorname{MANE}(\%)=\frac{1}{M} \sum_{i}^{M} \frac{\left|S R I_{i}-L S_{i}\right|}{L D_{i}} \cdot 100
$$

where $S R I_{i}$ and $L S_{i}$ are the $S R I$ value and the load shedding provided by Monte Carlo simulation at the $\mathrm{i}^{\text {th }}$ hour, respectively. $L D_{i}$ is the load demand at the $\mathrm{i}^{\text {th }}$ hour and $M$ is the total number of Monte Carlo simulation steps where there was probability of branch failure due to windstorm. It is emphasized that the simulation steps, 
where there was no probability of branch damages, were not considered in the computation of MANE as the load shedding (due to branch failures in previous steps) for these simulation steps could be predicted accurately.

\subsection{Test network and weather regions}

The test system was divided into four geographical (weather) regions as shown in Fig. 3 in order to account for the spatial impact of windstorms on the branches and towers of the transmission system. If the weather data across each transmission corridor was available with the spatial and temporal resolution desired, then they could be easily used by the proposed tool. When a transmission system crossed more than one region (e.g. branch $\mathrm{B} 11-\mathrm{B} 14)$, the worst weather condition was considered for determining the weather-dependent failure probability. The length of the branches between the buses was taken directly from Grigg et al. (1999) and it was assumed that there is one tower per $300 \mathrm{~m}$ of a branch connected in series. All the branches except those between B1-B2 and B6-B10 which are underground cables (see Fig. 3) are overhead lines. The underground branches are not affected by the windstorm and hence they were not considered in the impact modelling of the windstorm.

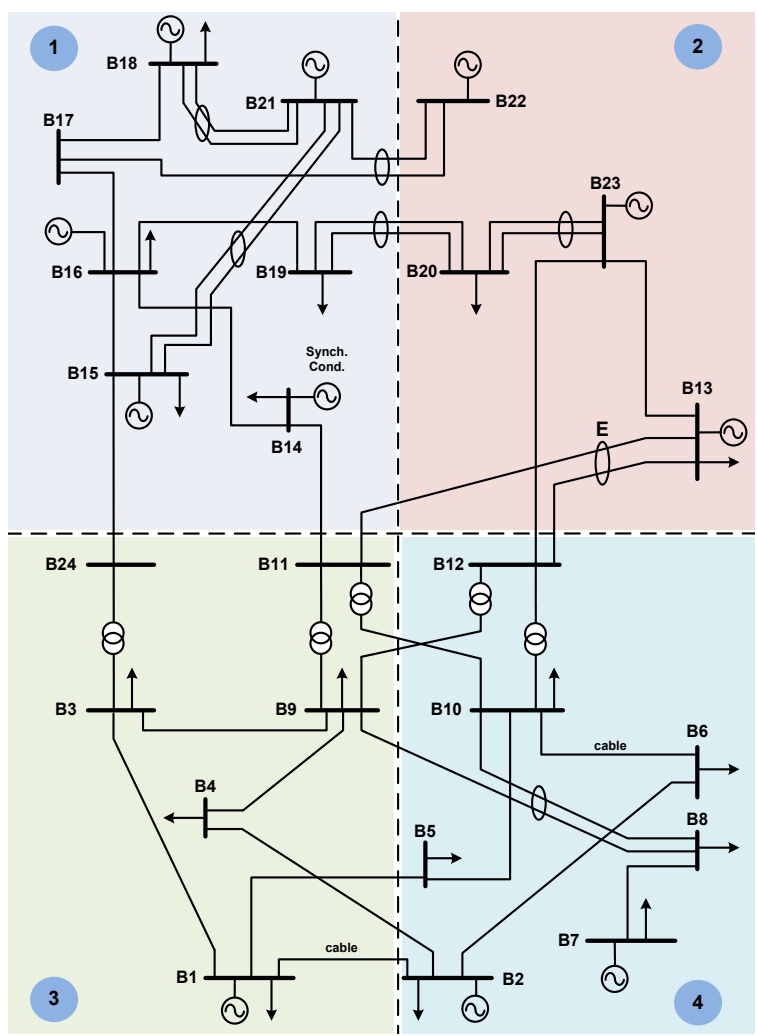

Fig. 3. Test system and weather regions. 


\subsection{Simulation data}

The fragility curves used to obtain the weather-dependent failure probabilities are shown in Fig. 4. The branch fragility curve was built based on a statistical analysis of weather-related branch outages in UK (Murray, \& Bell, 2014). The tower wind fragility curve is an example of the outputs of the Resilient Electricity Networks for Great Britain (RESNET) project, UK (Panteli, and Mancarella, 2015a). The sampling of fragility curves using evolving conditions within the time-series simulation procedure for determining the branch failure probabilities at each simulation step allows the systematic quantification of the spatiotemporal impact modelling of the windstorm on the individual components.

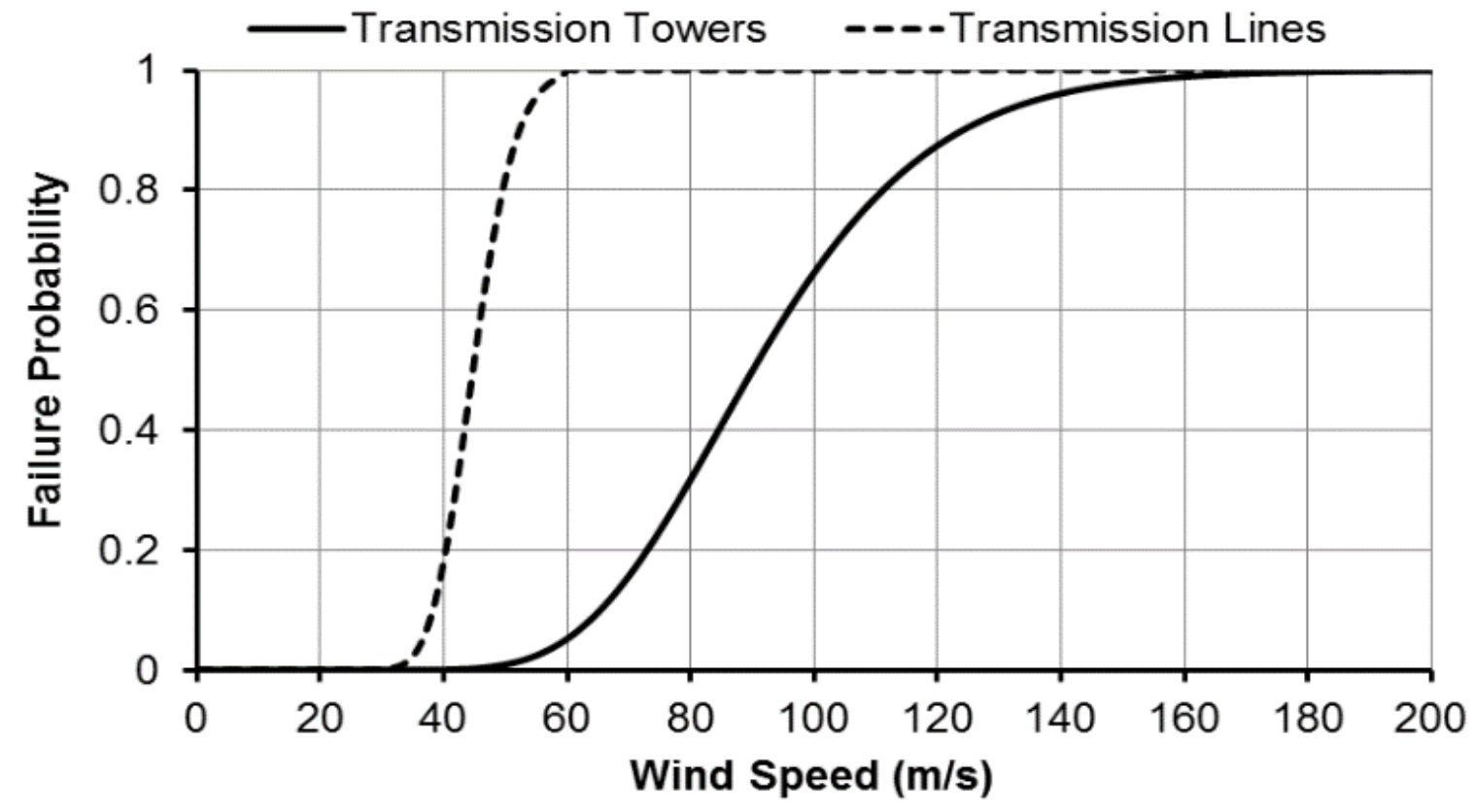

Fig. 4. Wind related fragility curves of transmission lines and towers.

Wind profiles representing hourly average wind speed were obtained by MERRA re-analysis (MERRA Re-analysis, 2016) and correspond to wind profiles at different locations in Great Britain. More specifically, 33 yearly wind profiles for the years 1979-2011 at four different location in Great Britain were obtained. The day with the maximum wind speed was selected from each year to demonstrate the effectiveness of the proposed methodology. More details on data are provided by Rienecker et al. (2011). MERRA-reanalysis provides data for six geographical locations across Great Britain. For simplicity and demonstration purposes, four weather regions were used for the test system in order to facilitate the illustration and presentation of the results. The

This article is protected by copyright. All rights reserved. 
weather conditions were assumed homogenous within each weather region and therefore the components within each region were exposed to the same wind conditions. This means that the fragility of the components was the same within each weather region. The wind data obtained by MERRA re-analysis have been scaled up using appropriate multiplication factors in order to model severe windstorms (e.g. with maximum wind speeds equal to $52 \mathrm{~m} / \mathrm{s}$, which is one of the highest wind speed that has been recorded in UK according to U.K. Met Office (2016)) that can be a threat for the network resilience. It is noted, that the hourly wind speed of each examined day (24 hours) is multiplied with the same multiplication factor at each simulation step.

\subsection{Simulation results}

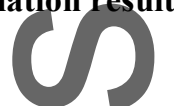

It was assumed that the normal failure probability of branches is $1 \%$. Each branch with a failure probability $P r_{b r}$ higher than $1 \%$ was considered vulnerable and was used to construct the possible failure scenarios. The threshold was selected in order to examine even the marginal impact of windstorm on power system components, and in turn on the risk and resilience of the whole system. $t h r s_{L H S}$ and $t h r S_{R E D}$ were selected to be 1000 and 100, respectively. The selected thresholds were determined after a large number of tests. The selected thresholds should enable the computation of SRI in a reasonable time, while SRI should capture the impact of the extreme event sufficiently. Therefore, if the number of possible failure scenarios was lower than 100, all the scenarios were considered for computing SRI. If the number of possible failure scenarios was between 100 and 1000 , only the scenarios reduction algorithm was applied to provide 100 representative scenarios. In case the number of possible failure scenarios was higher than 1000, LHS was applied to generate 1000 scenarios and then the scenario reduction algorithm was applied to provide 100 representative scenarios. Tables I and II present MANE for each geographical region depending on the number of possible failure scenarios, having considered zero and 10\% maximum forecast error for the wind speed of the next hour, respectively. Considering the forecast error, a uniformly distributed random number $m \sim U(-0.1,0.1)$ was generated for each simulation step (hour). The forecasted speed for the $\mathrm{i}^{\text {th }}$ hour was equal to the actual wind speed multiplied by the factor $\left(1+m_{i}\right)$. This forecasted wind speed was used to assess the impact of the event on the power system and compute $S R I$, while the actual wind speed was used to compute the load shedding $L S$. By comparing Tables I and II, it is observed that the increase of the forecast error leads to higher values of MANE. Consequently, the accuracy of the wind speed prediction has influence on the effectiveness of the proposed method. However, $S R I$ is able to capture the impact of windstorm on power system sufficiently, even in case of acceptable forecast errors. Moreover, as expected, there is the tendency to increase MANE with the increase of the number of 
possible failure scenarios. As the number of possible failure scenarios increases, the representative scenarios (after the application of scenario reduction algorithm) constitute an increasingly smaller percentage of the initial set of scenarios. Therefore, the estimation error tends to increase, as the number of possible scenarios increases. The branch failures in a region might have impact on another region and for this reason the number of failure scenarios is referred to the whole system. It is noted that the computation time of SRI at each simulation step was less than a minute which enables the online application of the model.

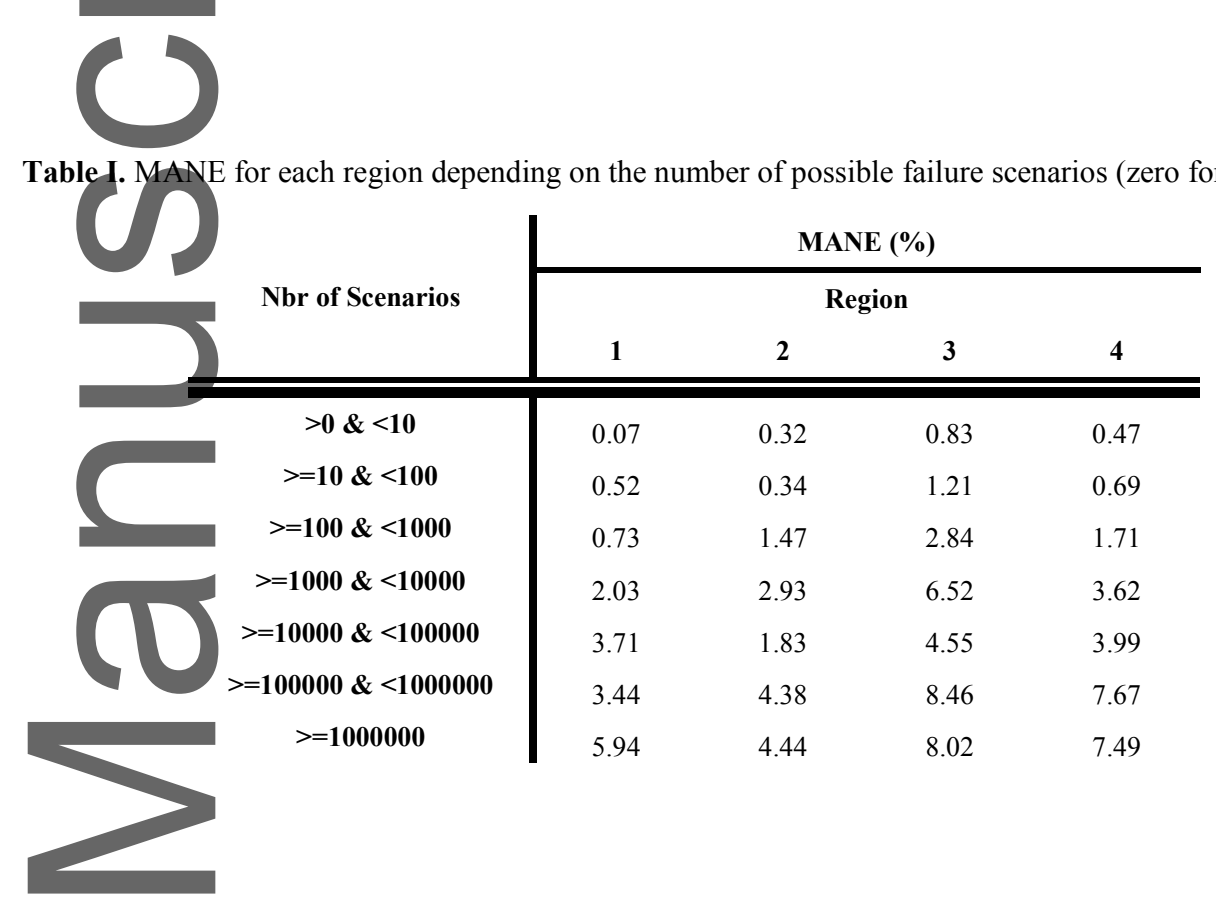

Table II. MANE for each region depending on the number of possible failure scenarios ( $10 \%$ maximum forecast error)

\begin{tabular}{|c|c|c|c|c|}
\hline \multirow{3}{*}{ Nbr of Scenarios } & \multicolumn{4}{|c|}{ MANE (\%) } \\
\hline & \multicolumn{4}{|c|}{ Region } \\
\hline & 1 & 2 & 3 & 4 \\
\hline$>0 \&<10$ & 0.48 & 0.67 & 0.93 & 0.70 \\
\hline$>=10 \&<100$ & 1.10 & 0.98 & 1.32 & 0.94 \\
\hline$>=100 \&<1000$ & 1.97 & 2.32 & 3.66 & 2.06 \\
\hline$>=1000 \&<10000$ & 2.82 & 3.66 & 7.30 & 4.74 \\
\hline$>=10000 \&<100000$ & 3.13 & 9.11 & 11.60 & 9.38 \\
\hline$=100000 \&<1000000$ & 6.05 & 10.02 & 14.44 & 11.71 \\
\hline$>=1000000$ & 7.56 & 11.72 & 13.01 & 12.93 \\
\hline
\end{tabular}

It is observed that for Region One, when the number of scenarios is between 10000 and 100000, the MANE value considering the wind forecast error is slightly smaller than the MANE value considering accurate prediction of the wind speed. The wind profiles used for Region One presented lower wind speeds in comparison with the wind profiles of the rest regions. For this reason, the deviations between Tables I and II for 
Region One increase less compared to the other regions. Furthermore, Region One was highly resistant, due to the existence of generators with high nominal capacity and double circuits in this region (Grigg et al., 1999). Therefore, the combination of the lower wind speeds and the resistant network led to a better estimation of extreme weather impact in this region, even considering wind speed forecast error. Taking into account that the forecast error had a little effect on the estimation of the impact on Region One, using the Sequential Monte Carlo simulation, a slightly better estimation resulted when the number of scenarios was between 10000 and 100000 despite the forecast error.

In addition, the maximum deviations between Tables I and II are detected for Region Two. This is due to the attributes of this region. In Region Two there were only two load buses (Buses 13 and 20). A generator was placed in bus 13 and the demand of this bus was satisfied even in an islanded state. Furthermore, bus 20 was connected directly through a double circuit with a generator bus and therefore the possibility of its demand not to be satisfied was low. Hence, a small change to SRI due to the wind forecast error resulted in high values of MANE.

It is noted that the proposed method assesses the impact of the extreme weather on power system and it is intended to be useful for supporting effective decision-making of preventive actions for mitigating the impact. Concerning a real-life application, the operator of the system that is responsible for applying the preventive actions, having in mind the estimation error of the proposed method, would apply the appropriate actions according to the security criteria to be fulfilled. For example, if the operator's objective is minimization of load shedding, the applied preventive actions should aim to the mitigation of a more severe impact than the estimated one by the method. Furthermore, in case of planning which loads to shed in a controllable way for satisfying the steady state constraints, the determined total load to shed should be higher than the estimated one. These critical decisions should take into account the estimated error of the proposed method.

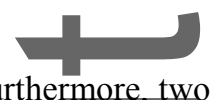

Furthermore, two windstorm scenarios were used for demonstrating the proposed SRI. Figs. 5 and 10 show the hourly time-series wind speed profiles for the four weather regions of the test system for Scenario One and Scenario Two, respeetively. These scenarios present different maximum wind speeds and regional time-series wind profiles. In Scenario One the maximum wind speed occurs in all regions almost simultaneously at the end of the day and the regional wind profiles progress in a similar way, while in Scenario Two the regional maximum wind speeds occur at different times. The maximum wind speed $(\approx 52 \mathrm{~m} / \mathrm{s})$ is detected in Scenario Two. The investigation of different scenarios allows the evaluation of the flexibility and effectiveness of the 
proposed $S R I$ when the test system is subject to windstorms of varying nature, in terms of both spatiotemporal domain and severity. However, it is noted that both Scenario One and Scenario Two are two iterations of the Sequential Monte Carlo simulation and therefore the overall effectiveness of the proposed method is not able to be illustrated only by these two scenarios. The overall effectiveness of the method is demonstrated by the results presented in Tables I and II.

In Figs. 6-9 and 11-14, the black dotted, dashed and straight lines represent the SRI with and without considering the wind forecast error and the load shedding $L S(\mathrm{MWh})$, respectively. As was already mentioned, the recorded load shedding was due to branches failures during Sequential Monte Carlo simulation. The red straight line with markers and the dash-dot line show the number of possible failure scenarios for each simulation step with and without considering the wind forecast error. The number of possible failure scenarios depends on the number of vulnerable branches at each simulation step. As mentioned, the number of possible scenarios refers to the whole system and therefore for the same scenario, the graph of the number of the possible failure scenarios is the same for each region. It can be seen that despite the large number of possible failure scenarios in many simulation steps, the proposed methodology is capable of providing the operator useful information for the possible amount of load shedding and the degradation of the system state at each simulation step, even in the case of the wind forecast error.

It is observed that when the wind speed was less than $30 \mathrm{~m} / \mathrm{s}$ there was no possibility of branch failures, as according to fragility curves the probability of a windstorm to damage the components of a system becomes higher than zero for wind speeds higher than $30 \mathrm{~m} / \mathrm{s}$. Moreover, close to zero values of $S R I$ and load shedding may occur in simulation steps with a very high number of possible failure scenarios. This happens because each branch with a failure probability higher than $1 \%$ was considered as vulnerable and it participated in the generation of possible failure scenarios. When the speed in a region exceeds or is close to $30 \mathrm{~m} / \mathrm{s}$, the probability for each branch to fail is close to $1 \%$, which results in a huge number of possible failure scenarios with a very low probability and thus the $S R I$ and load shedding take values close to zero. Moreover, if branch failures have not occurred in previous hours, the system may be able to withstand the stress imposed by the windstorm without any load shedding. On the other hand, there were simulation steps where the SRI assumed high values when the number of scenarios was lower, because the effect of these scenarios in terms of load shedding was higher, mainly due to the branch failures in previous hours and therefore the degraded state of the system. The higher values of $S R I$ and load shedding were noticed at hours when the wind speeds reached their maximum, because the probability of a branch damage takes higher values. In addition, the branch damages of previous 
hours have led the system in a degraded state that it was not able to absorb the stress. Consequently, the load shedding depends on wind speed, system topology and component reliability characteristics, and the relevant previous history of the system damage during the extreme event. During the progress of the wind profiles the wind speed may increase, while the number of possible failure scenarios decreases. This happens because during previous hours some branches have already failed and thus they were not included in the vulnerable branches and the generation of possible failure scenarios for the next hours. Hence, the number of possible failure scenarios depends on the wind speed and the branch failures in previous hours.

There were simulation steps where SRI coincides with load shedding. This happens either because there was no probability of branch failures or the possible scenarios did not have any impact on a specific region. In both cases, the loadshedding was due to branch damages in previous hours. For example, Region One in Scenario Two was not affected by the branch failures in the other regions after the $13^{\text {th }}$ hour and the load shedding in this region was due to branch failures in previous hours. The winds in the other regions, that reached their maximum speed almost simultaneously when the wind speed in Region One has fallen around to $30 \mathrm{~m} / \mathrm{s}$, did not have impact on Region One. Furthermore, it is observed that in Scenario Two the wind in Region One did not affect the other regions in terms of load shedding.

By comparing $S R I$ with and without considering the wind forecast error, it is observed that the $S R I$ without forecast error, estimates with higher accuracy the load shedding for the larger percentage of the simulation steps. However, even in the case of the wind forecast error, the proposed method was able to estimate the impact of the extreme event satisfactorily. The higher deviations between $S R I$ with and without considering wind forecast error were detected during hours with a high number of possible failure scenarios that is also depicted in the results of Tables I and II. Concerning the number of possible failure scenarios, the higher deviations were detected for wind speeds close to $30 \mathrm{~m} / \mathrm{s}$, where the slope of the line fragility curve increases and the forecast error may lead to false identification of vulnerable lines. A positive absolute error may lead to the identification of a line as vulnerable, which is not according to the actual wind speed, while a negative absolute error may lead to the opposite results. Note that each branch with a failure probability $P r_{b r}$ higher than $1 \%$ was considered as vulnerable. When the wind speeds were not close to $30 \mathrm{~m} / \mathrm{s}$, the number of possible failure scenarios were identical, as the same lines were identified as vulnerable with and without considering the forecast error. It is noted, that the identical number of failure scenarios does not mean that the possible failure scenarios had the same probability, as the failure probability of a vulnerable line depended on the wind speed. 
By comparing Scenario One and Scenario Two, it is noted that in Scenario One the maximum number of possible failure scenarios was larger, despite the maximum wind speed was detected in Scenario Two. The reason is that in Scenario One the four regional wind profiles followed similar progress and reached their maximum wind speed almost simultaneously, while in Scenario Two the wind speed in Region Four has reached its maximum value several hours before the other regions reached their maximum wind speeds. Moreover, in Scenario Two higher values of load shedding are observed, simply because the wind speeds took higher values in this scenario. In both scenarios, Region Two had zero load shedding and close to zero SRI. As has already mentioned, in this region there were only two load buses. A generator was placed in bus 13 and the demand of this bus was satisfied even in an islanded state. Furthermore, bus 20 was connected directly through a double circuit with a generator bus and therefore the possibility of its demand not to be satisfied was low. Due to close to zero load shedding and close to zero $S R I$, a small change to SRI in Region Two, because of the wind forecast error, resulted to high deviations of MANE. This is observed by comparing Tables I and II, where the highest deviations between the tables are detected in Region Two.

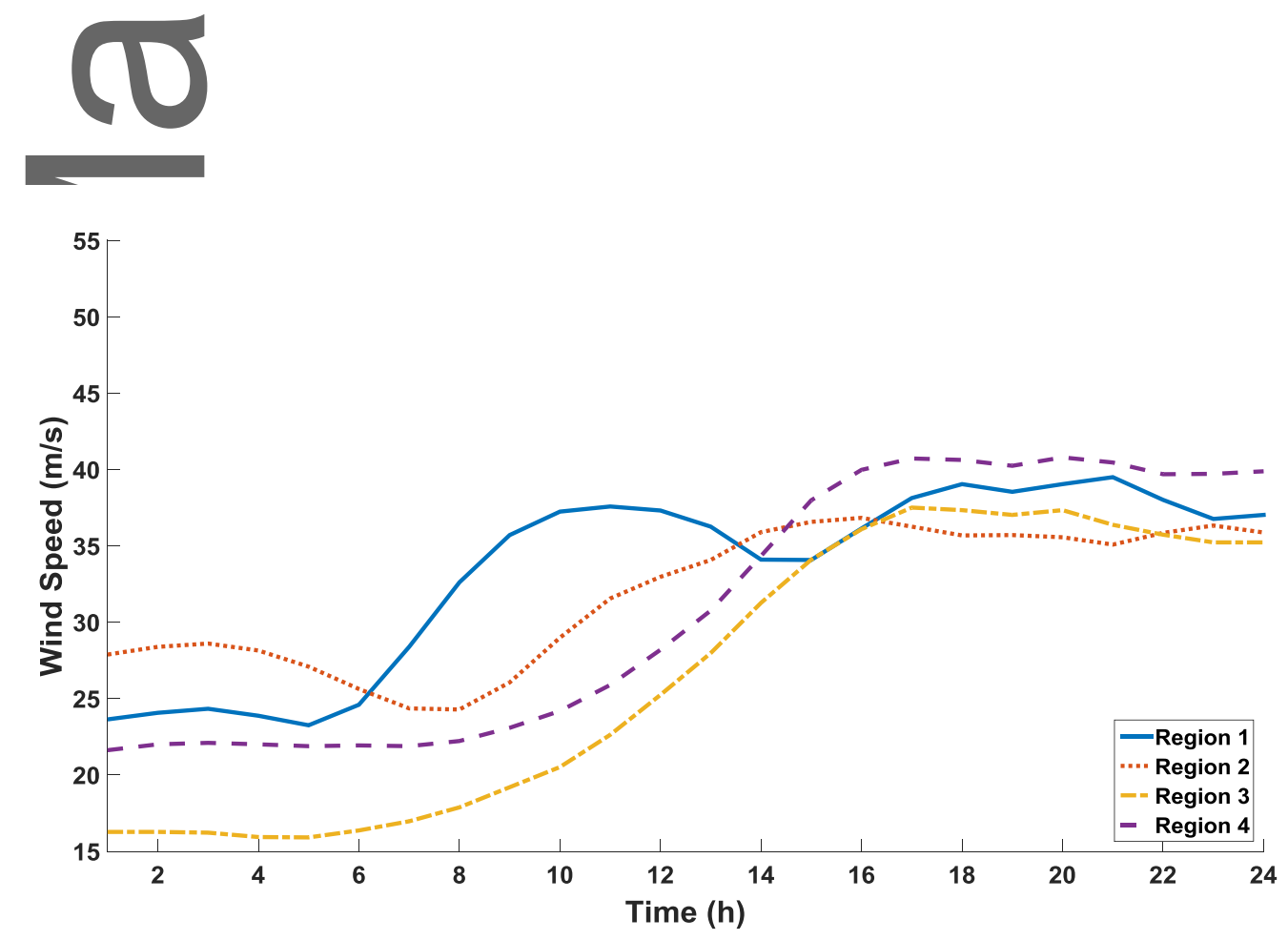

Fig. 5. Regional time-series wind profiles of Scenario One. 


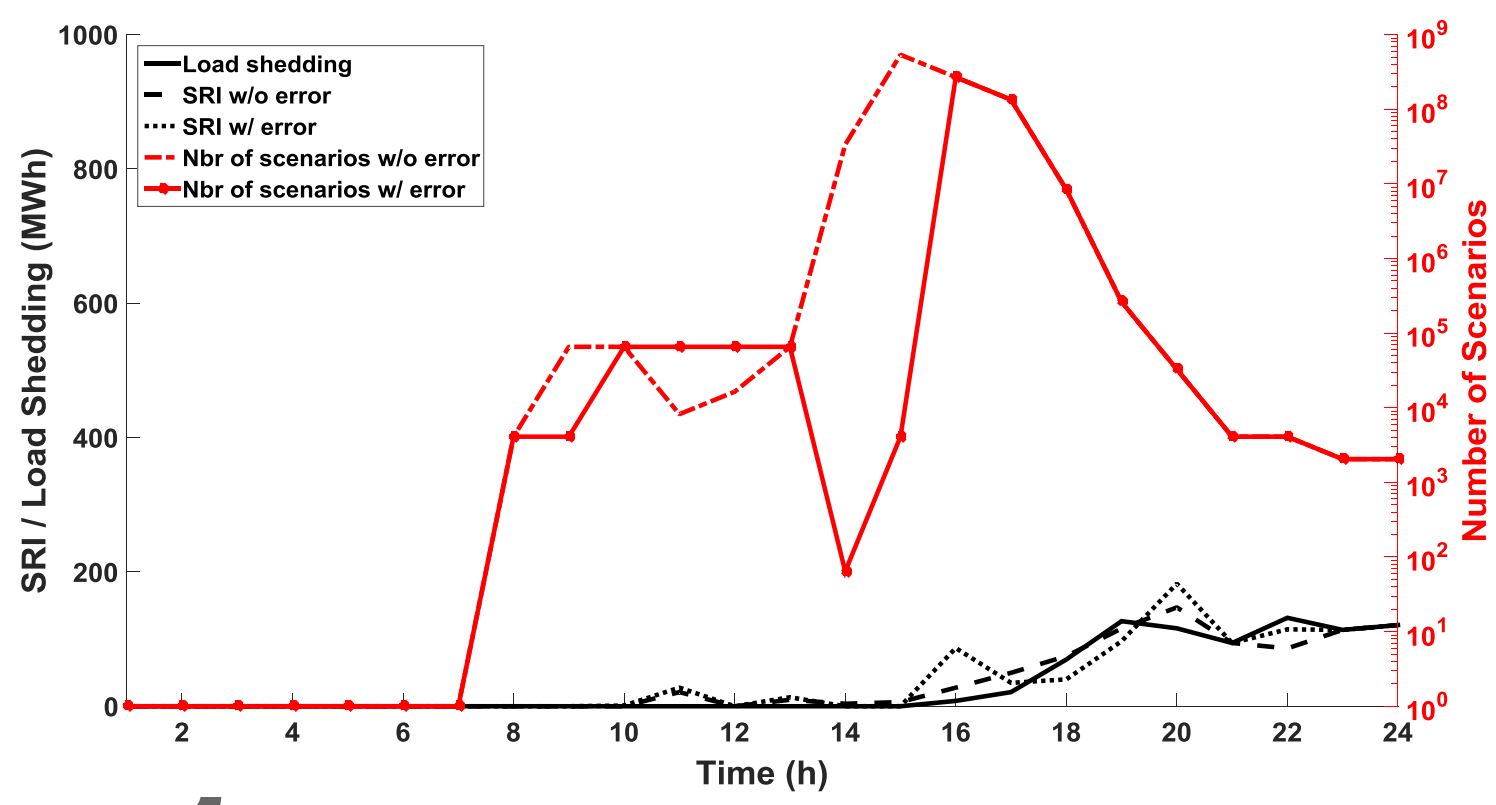

Fig. 6. $S R I$ with and without forecast error, load shedding, number of possible failure scenarios with and without forecast error for Region One (Scenario One).

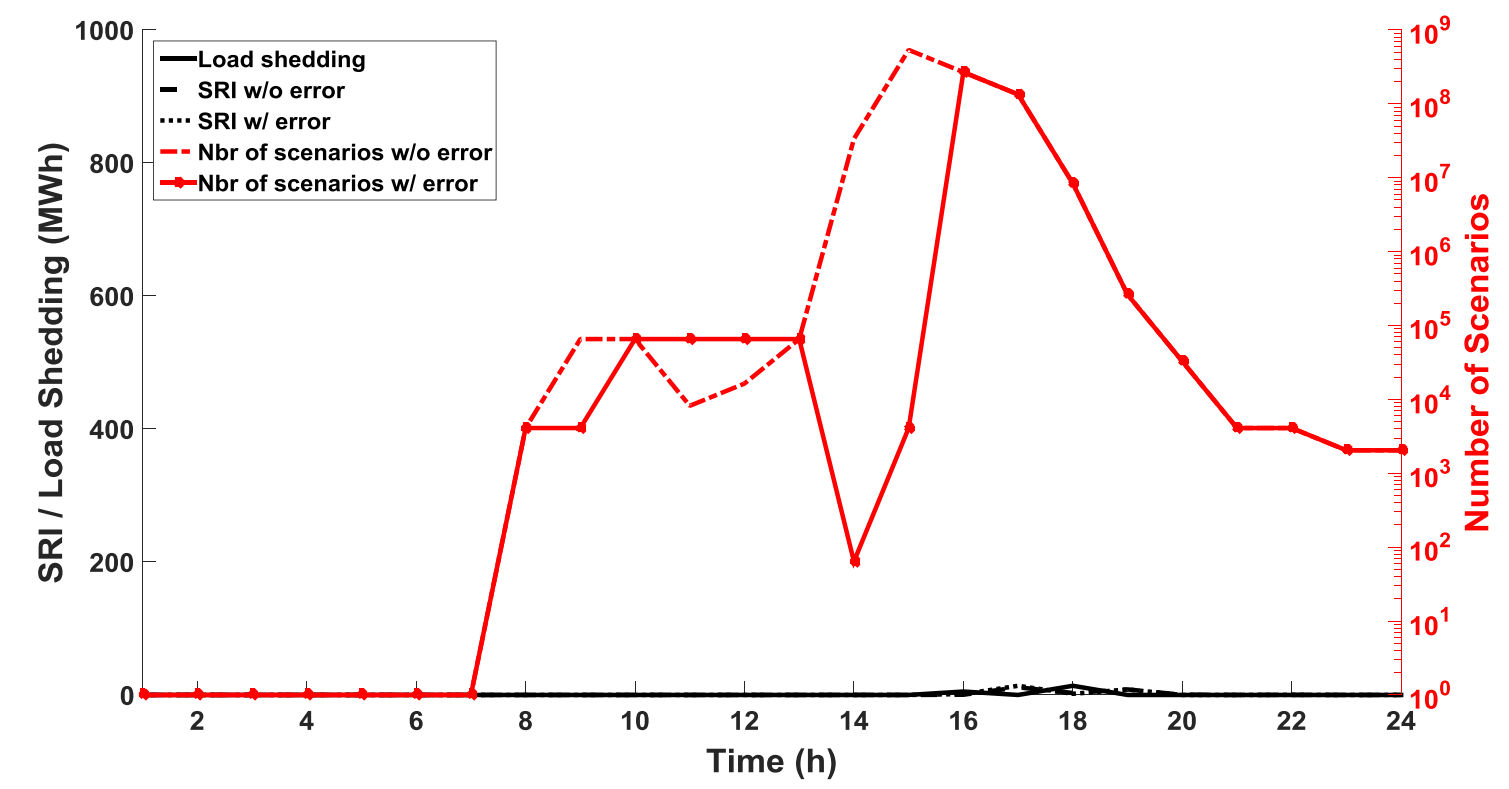

Fig. 7. SRI with and without forecast error, load shedding, number of possible failure scenarios with and without forecast error for Region Two (Scenario One). 


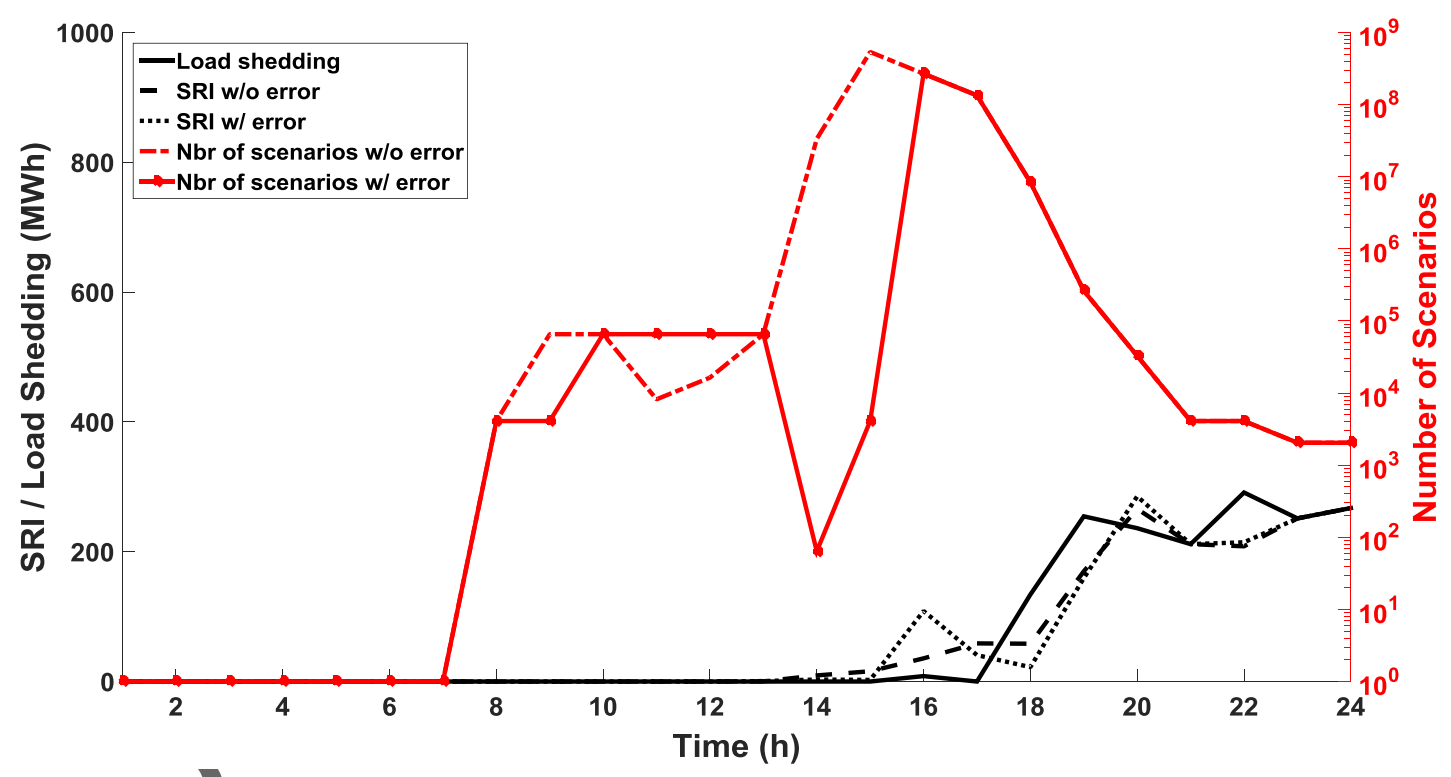

Fig. 8. $S R I$ with and without forecast error, load shedding, number of possible failure scenarios with and without forecast error for Region Three (Scenario One).
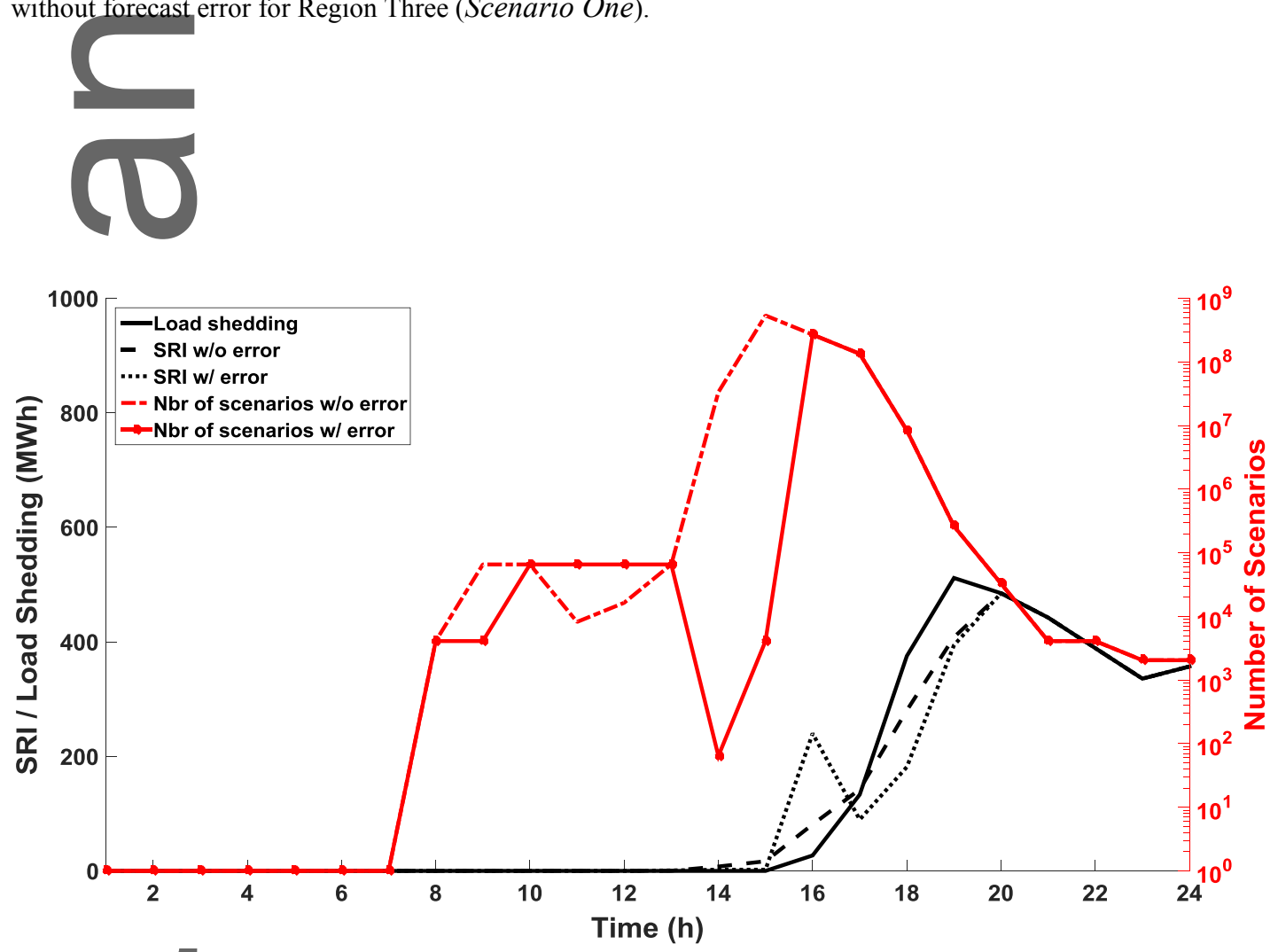

Fig. 9. SRI with and without forecast error, load shedding, number of possible failure scenarios with and without forecast error for Region Four (Scenario One). 


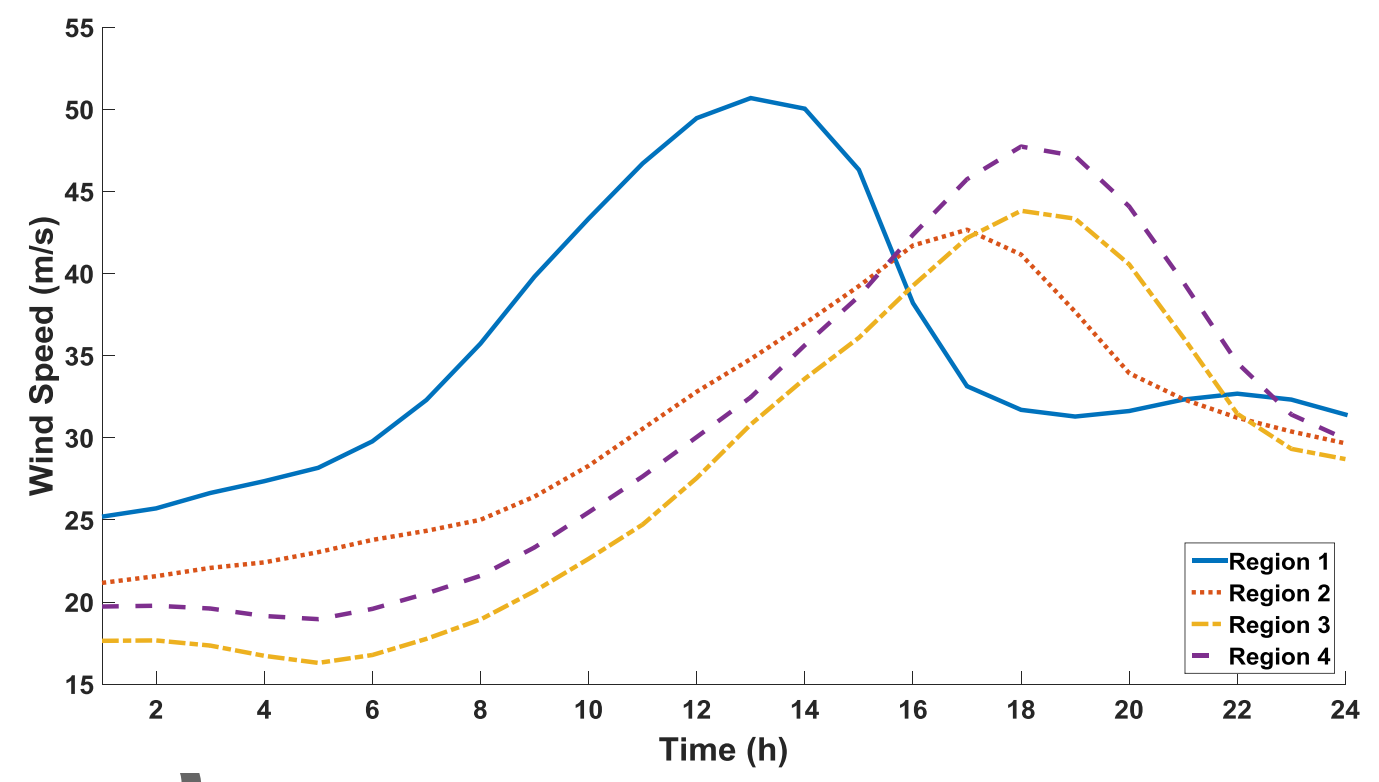

Fig. 10. Regional time-series wind profiles of Scenario Two.

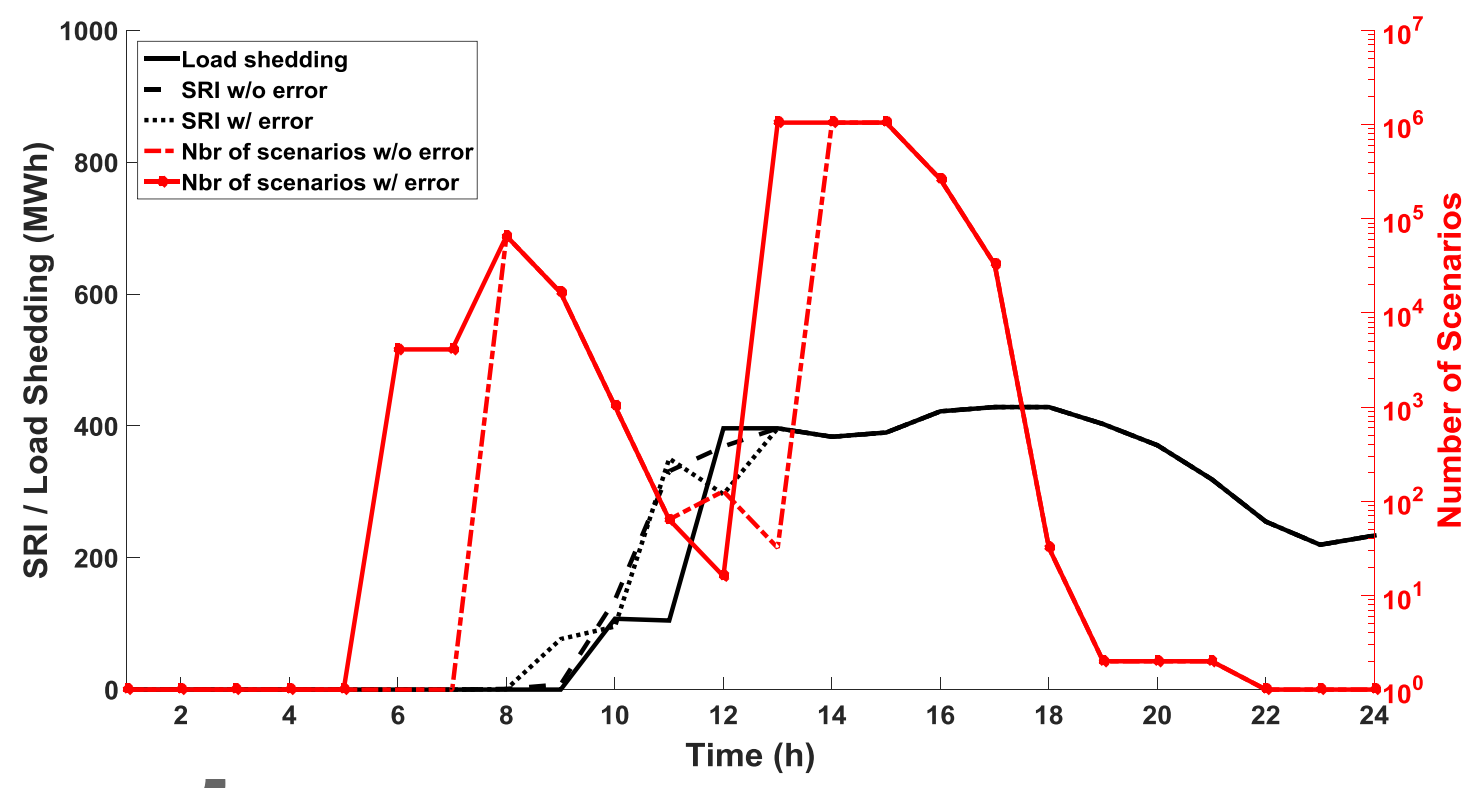

Fig. 11. SRI with and without forecast error, load shedding, number of possible failure scenarios with and without forecast error for Region One (Scenario Two). 


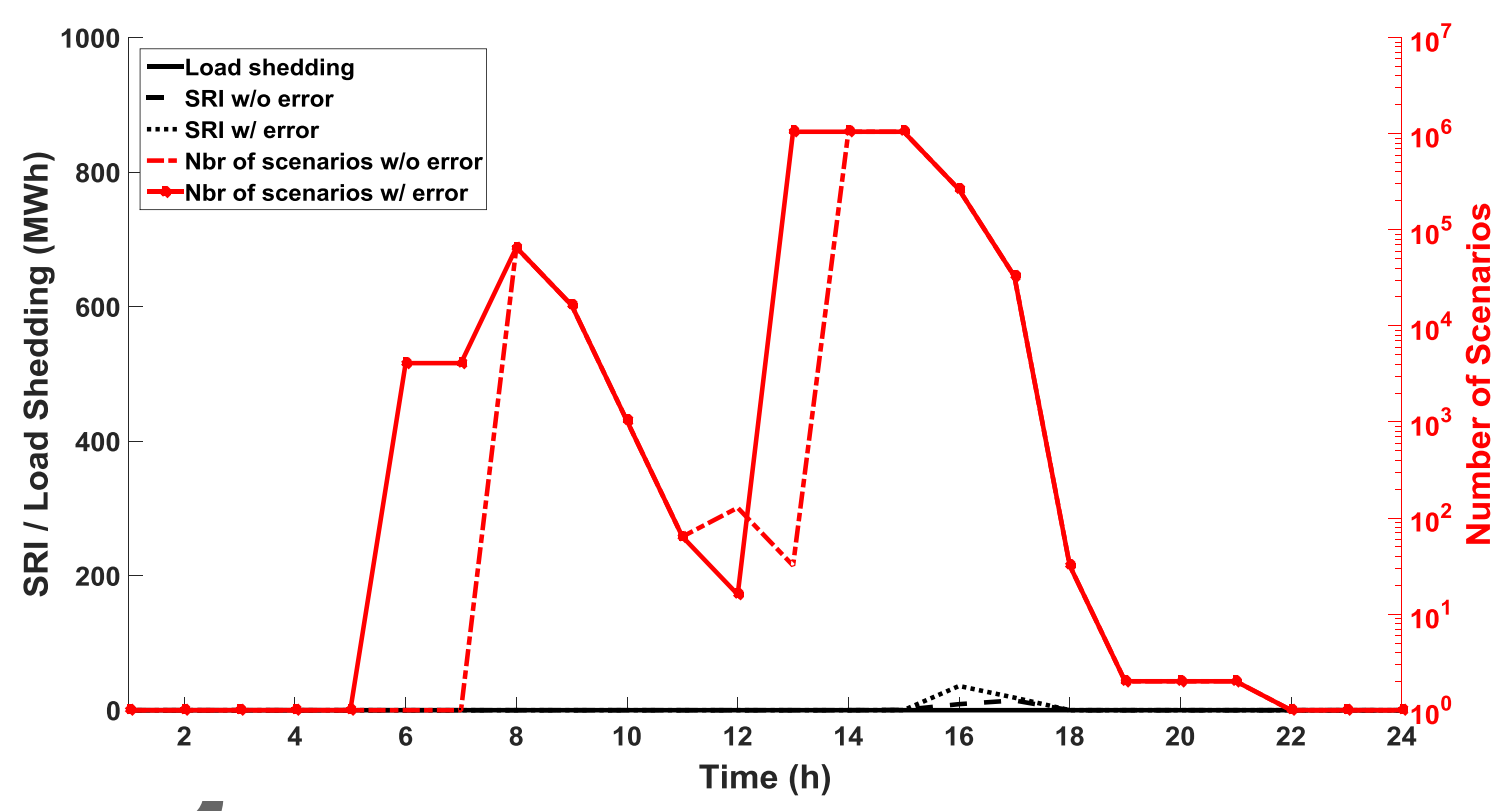

Fig. 12. $S R I$ with and without forecast error, load shedding, number of possible failure scenarios with and without forecast error for Region Two (Scenario Two).
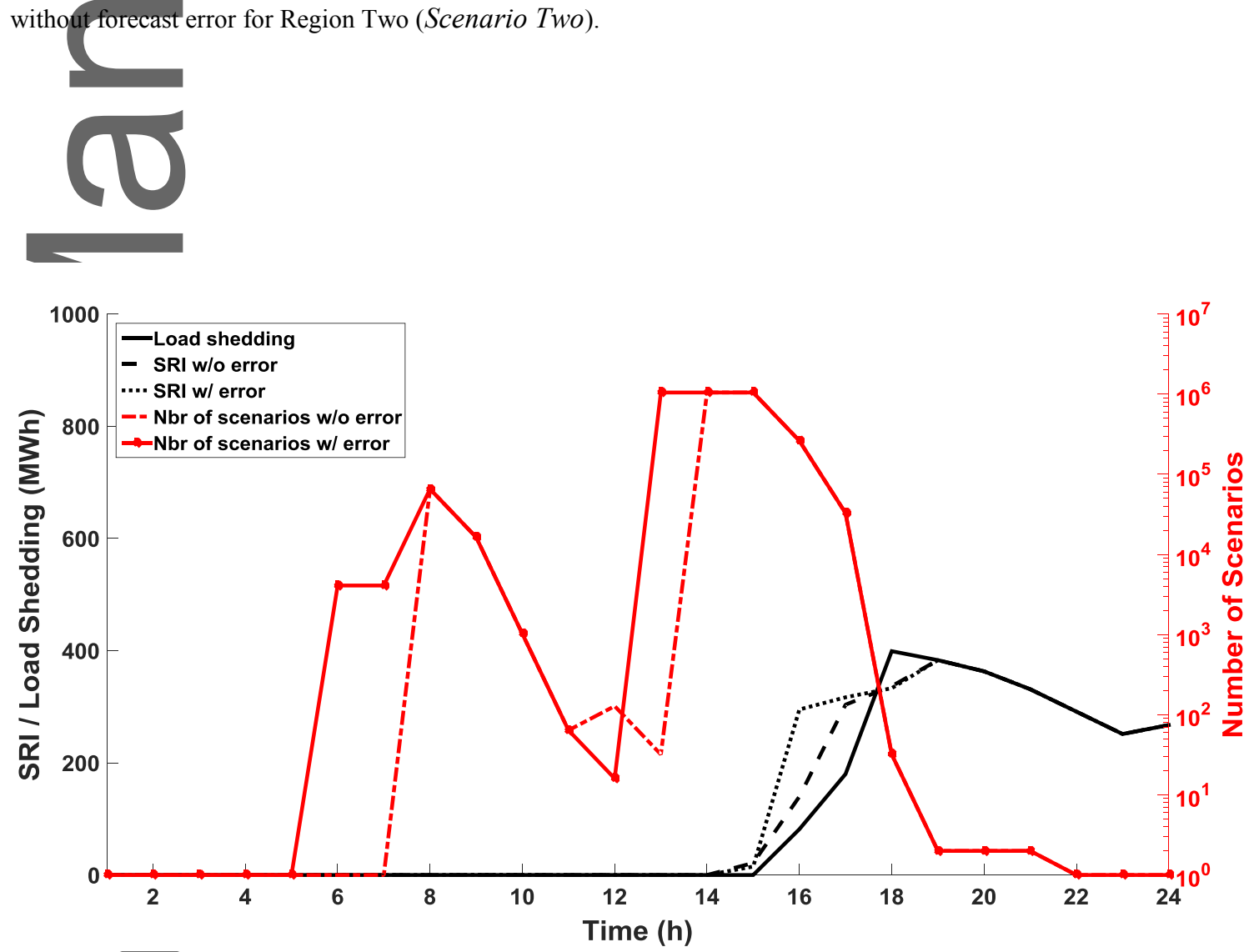

Fig. 13. SRI with and without forecast error, load shedding, number of possible failure scenarios with and without forecast error for Region Three (Scenario Two). 


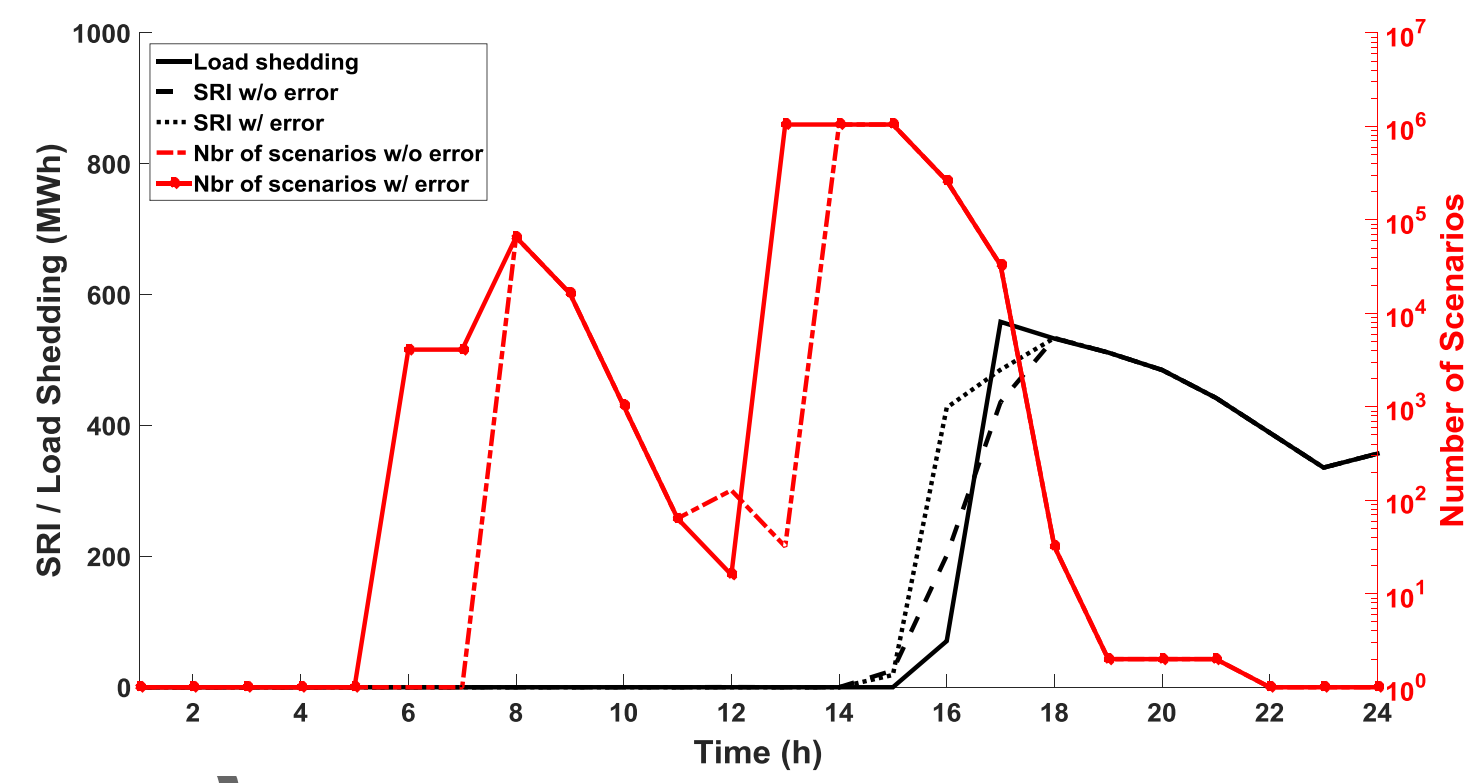

Fig. 14. SRI with and without forecast error, load shedding, number of possible failure scenarios with and without foreeast error for Region Four (Scenario Two).

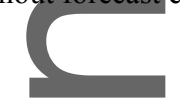

In general, it can be appreciated from Figs. 5-14 that the estimated SRI is capable of reflecting the pattern of the regional wind profiles, meaning that high values of SRI occur around or at the maximum of the wind profiles. Therefore, if the severity and location of the upcoming weather event can be accurately predicted or with acceptable forecast errors, the proposed $S R I$ is capable of spatially and temporally quantifying the risk imposed to the network by the extreme weather event in an adaptive and preventive mode. It thus allows, to implement potential effective and timely remedial actions (e.g., power rerouting or defensive islanding (Panteli, Trakas, Mancarella, \& Hatziargyriou (2016)) for mitigating the impact on the transmission network and plan appropriately which loads to shed in a controllable way, in order to maintain the uninterrupted supply of critical loads. Lower time and spatial resolution than one hour and more than four weather regions can be incorporated in the proposed tool, if accurate weather data at the desired resolution are available.

\section{CONCEUSION}

The present paper has presented an online risk analysis for evaluating the spatiotemporal impacts of extreme exents on transmission networks. A Severity Risk Index (SRI) has been proposed, which takes into account the probability and impact of possible branch damages that may occur during the event, with the application of extreme windstorm events. It has been demonstrated that $S R I$ is capable of capturing the spatial risk imposed to the transmission network at various geographical regions by the severe weather event, at an 
hourly basis. The proposed method is also capable of using lower temporal and spatial resolutions, if desired. It thus provides the system operator with a probabilistic tool to assess the system resilience. The computation time of SRI (i.e., less than a minute for each hour) enables the real-time support of mitigation decisions, such as power rerouting or the application of defensive islanding. Moreover, it allows the appropriate planning of which loads to shed in a controllable way, in order to maintain the steady state security of the system. Latin Hypercube Sampling (LHS) and Backward Reduction Algorithm were used to produce a computationally manageable number of preserved scenarios for computing $S R I$ at every simulation step and enable the online application of the proposed model.

\section{(}

The proposed method has been applied on the IEEE 24-bus Reliability Test System. A Sequential Monte Carlo-based simulation engine was used to run the time-series simulations and record the necessary information, e.g. vulnerable components and amount of load shedding, to estimate $S R I$ and evaluate the effectiveness of the proposed method. For each geographical region, the Mean Absolute Normalized Error (MANE) was computed and the simulation results clearly demonstrate the effectiveness of the method. The proposed method has been evaluated considering zero and $10 \%$ maximum forecast error for the wind speed of the next hour. It has been shown that the method was able to estimate the impact of the extreme event on the system with sufficiently accuracy even in the case of acceptable forecast errors. Two windstorm scenarios were used for illustrating the proposed SRI, with different wind profiles and severity. It has been demonstrated that even during the hours with high number of possible failure scenarios or wind speeds, the proposed method managed to obtain the expected load shedding sufficiently. In addition, it has been observed that the load shedding not only depends on the wind speed of each hour but also on the nature of wind speed time-series on each region and therefore the branch failures of previous hours.

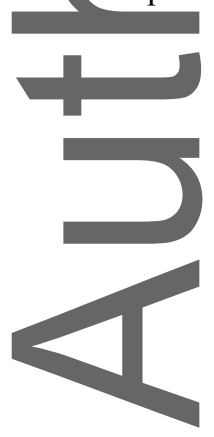




\section{REFERENCES}

Bhat, R., \& Meliopoulos, A. (2016). Probability of distribution network pole failures under extreme weather conditions. Clemson University Power Systems Conference (PSC) (pp. 1-6). Clemson, SC.

Chen, X., Wu, Y., \& Lou, S. (2011). Risk assessment for power system static security based on fuzzy modeling of weather conditions. IEEE Power Engineering and Automation Conference (pp. 367-371). Wuhan.

Ciapessoni, E., Cirio, D., Grillo, S., Massucco, S., Pitto A., \& Silvestro F. (2013). An Integrated Platform for Power System Security Assessment Implementing Probabilistic and Deterministic Methodologies. IEEE Systems Journal, 7, 845-853.

Eidinger, J. M., \& Kempner, L. Jr. (2012). Reliability of Transmission Towers under Extreme Wind and Ice Loading. 44th General Session of CIGRÉ B2-211.

Espinoza, S., Panteli, M., Mancarella, P., \& Rudnick, H. (2016). Multi-phase assessment and adaptation of power systems resilience to natural hazards. Electric Power Systems Research, 136, 352-361.

Grigg, C., Wong, P., Albrecht, P., Allan, R., Bhavaraju, M., Billinton, R., et al. (1999). The IEEE Reliability Test System-1996. A report prepared by the Reliability Test System Task Force of the Application of Probability Methods Subcommittee. IEEE Transactions on Power Systems, 14(3), 1010-20.

Growe-Kuska, N., Heitsch, H., \& Romisch, W. (2003). Scenario reduction and scenario tree construction for power management problems. IEEE Bologna Power Tech Conference Proceedings.

Guikema, S. D., Quiring, S. M., \& Han, S.-R. (2010). Prestorm Estimation of Hurricane Damage to Electric Power Distribution Systems. Risk Analysis, 30, 1744-1752.

Guikema, S. D., Nateghi, R., Quiring, S. M., Staid, A., Reilly, A. C., \& Gao M. (2014). Predicting Hurricane Power Outages to Support Storm Response Planning. IEEE Access, 2, 1364-1373.

Han, S.-R., Guikema, S. D., \& Quiring, S. M. (2009). Improving the Predictive Accuracy of Hurricane Power Outage Forecasts Using Generalized Additive Models. Risk Analysis, 29, 1443-1453.

Han, S.-R., Guikema, S. D., Quiring, S. M., Lee, KH, Davidson, R., \& Rosowsky D. (2009). Estimating the spatial distribution of power outages during hurricanes in the Gulf Coast region. Reliability Engineering and System Safety, 94(2), 199-210.

He, J., Wanik, D. W., Hartman, B. M., Anagnostou, E. N., Astitha, M. \& Frediani, M. E. B. (2016). Nonparametric Tree-Based Predictive Modeling of Storm Outages on an Electric Distribution Network. Risk Analysis, 37, 441-458.

Heitsch, H., \& Römisch W. (2003). Scenario reduction algorithms in stochastic programming. Computational. Optimization and Applications, 24,187-206.

Li G., Zhang, P., Luh, P.B., Li, W., Bie, Z., Serna, C., \& Zhibing, Z. (2014). Risk Analysis for Distribution Systems in the Northeast U.S. Under Wind Storms. IEEE Transactions on Power Systems, 29(2), 889-98.

Liu, H., Davidson, R.A., \& Apanasovich, T.V. (2008). Spatial generalized linear mixed models of electric power outages due to hurricanes and ice storms. Reliability Engineering \& System Safety, 93(6), 897-912.

Liu, Y., \& Singh, C. (2011). A Methodology for Evaluation of Hurricane Impact on Composite Power System Reliability. IEEE Transactions on Power Systems, 26(1), 145-52.

Mckay, M.D., Beckman, R.J., \& Conover, W.J. (2000). A Comparison of Three Methods for Selecting Values of Input Variables in the Analysis of Output from a Computer Code. Technometrics, 42(1), 55. 
MERRA Re-analysis (2016). Available at: http://gmao.gsfc.nasa.gov/

Murray, K., \& Bell, K.R.W. (2014). Wind related faults on the GB transmission network. International Conference on Probabilistic Methods Applied to Power Systems (PMAPS) (pp. 1-6). Durham.

Nateghi, R., Guikema, S., \& Quiring, S. M. (2014). Power Outage Estimation for Tropical Cyclones: Improved Accuracy with Simpler Models. Risk Analysis, 34, 1069-1078.

National Infrastructure Advisory Council. (2010). A framework for establishing critical infrastructure resilience goals, Washington, DC, USA.

Ouyang, M., \& Dueñas-Osorio, L. (2014). Multi-dimensional hurricane resilience assessment of electric power systems. Structural Safety, 48, 15-

Panteli, M., \& Mancarella, P. (2015a). Influence of extreme weather and climate change on the resilience of power systems: Impacts and possible mitigation strategies. Electric Power Systems Research, 127, 259-70.

Panteli, M., \& Mancarella, P. (2015b). Modeling and Evaluating the Resilience of Critical Electrical Power Infrastructure to Extreme Weather Events. IEEE Systems Journal, 11(3), 1733-1742.

Panteli, M., \& Mancarella, P. (2015c). The Grid: Stronger, Bigger, Smarter?: Presenting a Conceptual Framework of Power System Resilience. IEEE Power and Energy Magazine, 13(3), 58-66.

Panteli, M., Pickering, C., Wilkinson, S., Dawson, R., \& Mancarella, P. (2016). Power System Resilience to Extreme Weather: Fragility Modelling, Probabilistic Impact Assessment, and Adaptation Measures, IEEE Transactions on Power Systems, 32 (5), $3747-3757$.

Panteli, M., Trakas, D.N., Mancarella, P., \& Hatziargyriou, N.D. (2016). Boosting the Power Grid Resilience to Extreme Weather Events Using Defensive Islanding. IEEE Transactions on Smart Grid, 7(6), 2913-2922

Quiring, S. M., Zhu, L., \& Guikema, S. D. (2011). Importance of soil and elevation characteristics for modeling hurricane-induced power outages. Nat Hazards, 58, 365-390.

Rienecker, M.M., Suarez, M.J., Gelaro, R., Todling, R., Bacmeister, J., Liu, E., Bosilovich, M.G., Schubert, S.D., Takacs, L., Kim, G., Bloom, S., Chen, J., Collins, D., Conaty, A., da Silva, A., Gu, W., Joiner, J., Koster, R.D., Lucchesi, R., Molod, A., Owens, T., Pawson, S., Pegion, P., Redder, C.R., Reichle, R., Robertson, F.R., Ruddick, A.G., Sienkiewicz, M., \& Woollen J. (2011). MERRA: NASA's ModernEra Retrospective Analysis for Research and Applications. J. Climate, 24, 3624-3648.

Song, X., Wang, Z., Xin, H., \& Gan, D. (2013). Risk-based dynamic security assessment under typhoon weather for power transmission system. IEEE PES Asia-Pacific Power and Energy Engineering Conference (APPEEC) (pp. 1-6). Kowloon.

U.K. Met Office (2016). Beaufort Wind Force Scale. Available at http://www.metoffice.gov.uk/learning/wind/windiest-place-in-uk.

Vincent, P., Huet, G., Charbonneau, M., Guillbault, P., Lapointe, M., \& Banville, D. (2004). Testing and numerical simulation of overhead transmission lines dynamics under component failure conditions. 40th General Session of CIGRÉ, B2-308.

Wanik, D.W., Anagnostou E.N., Hartman, B.M., Frediani, M.E.B, \& Astitha, M. (2015). Storm outage modeling for an electric distribution network in Northeastern USA. Nat Hazards, 79, 1359-1384.

Xie, L., Bao, S., Pietrafesa, L. J., Foley, K., \& Fuentes, M. (2006). A real-time hurricane surface wind forecasting model: formulation and verification. Monthly Weather Review, 134(5), 1355-1370.

Xu, L., \& Brown, R.E. (2008). A hurricane simulation method for Florida utility damage and risk assessment. IEEE Power and Energy Society General Meeting - Conversion and Delivery of Electrical Energy in the 21st Century (pp. 1-7). Pittsburgh, PA. 


\section{University Library}

\section{- M M N E R VA A gateway to Melbourne's research publications}

Minerva Access is the Institutional Repository of The University of Melbourne

Author/s:

Trakas, DN;Panteli, M;Hatziargyriou, ND;Mancarella, P

Title:

Spatial Risk Analysis of Power Systems Resilience During Extreme Events

Date:

2019-01-01

Citation:

Trakas, D. N., Panteli, M., Hatziargyriou, N. D. \& Mancarella, P. (2019). Spatial Risk Analysis of Power Systems Resilience During Extreme Events. RISK ANALYSIS, 39 (1), pp.195-211. https://doi.org/10.1111/risa.13220.

Persistent Link:

http://hdl.handle.net/11343/284704 